\title{
Assesment of Some Heavy Metals in Water, Sediments And Fish During 2013 in Lake Quaron Protected Area, Fayoum, Egypt.
}

\author{
Lamia I. Mohamedien*, Medani G.G., Gamal Eldien M.A.** \\ and Hammad M. H. $* * *$
}

Marine Ecology Department, Suez Branch National Institute of Oceanography and Fisheries*, Department of Wildlife Management, Faculty of Veterinary Medicine, Suez Canal University**, and Egyptian Environmental Affairs Agency***

\begin{abstract}
:
Heavy metal concentrations in the environment are of great concern due to their serious effects through the food chain on animal and human health. In this study, 26 water samples and 26 sediment samples were collected from the 13 site in Lake Quaron Protected area during summer and spring seasons with 40 fish samples of four species (Atherinasp. Tilapia zillii, Mugil cephalus and Solea vulgaris) were examined for evaluation of heavy metals pollution. The results revealed that the annual average concentrations $(\mu \mathrm{g} / \mathrm{l})$ of the heavy metals in water samples were in the following order; $\mathrm{Zn}>\mathrm{Fe}>\mathrm{Ni}>\mathrm{Mn}>\mathrm{Cu}>\mathrm{Pb}>\mathrm{Cd}$ and within the permissible limits, the annual average concentrations $(\mu \mathrm{g} / \mathrm{g})$ of the heavy metals in surface sediment samples were in the following order; $\mathrm{Fe}>\mathrm{Mn}>\mathrm{Zn}>\mathrm{Pb}>\mathrm{Cu}>\mathrm{Ni}>\mathrm{Cd}$ and within the permissible limits of Sediment quality guide lines except $\mathrm{Cu}$ was higher and the levels of heavy metals in fish were in the following order ;Atherina sp.> Tilapia zillii $>$ Mugil cephalus $>$ Solea Vulgaris. The evaluation of examined heavy metals pollution in Lake Quaron showed that Lake Quaron protected area suffers from a serious pollution problem.

Key words: Heavy metals, Lake Quaron
\end{abstract}




\section{Introduction}

Lake Quaron considered one of oldest lake in Egypt and it was declared as protected area in 1989 by the decision No. 348 of the Prime Minister for protecting the geological, biological and archaeological diversity that found in the area.

The Lake is in the Fayoum Province, $40 \mathrm{~km}$ in length, $5.7 \mathrm{~km}$ in width and 34 to $43 \mathrm{~m}$ below sea level (with a mean depth of $4.2 \mathrm{~m}$.). Lake Quaron receives the agricultural and sewage drainage through a system of twelve drains, most of the drainage water reaches the lake by two main drains .ElBatts and El-Wadi, whereas there are minor drains discharged its drainage water into the lake by mains of hydraulic pumps but in small amounts (Authman and Abbas 2007).

The term heavy metal refers to any metallic chemical element that has a relatively high density and is toxic or poisonous at low concentrations. Aquatic organisms absorb the pollutants directly from water and indirectly from food chains. Some of the toxic effects of heavy metals on fishes and aquatic invertebrates are; reduction of the developmental growth, increase of developmental anomalies, reduction of fishes survival especially at the beginning of exogenous feeding or even cause extinction of entire fishes population in polluted reservoirs (Khayatzadeh and Abbasi 2010). This work aimed to detect the heavy-metal levels in Lake Quaron through water, sediment and fish samples.

\section{Material and methods}

Five liter of water and $100 \mathrm{~g}$ of sediment samples were collected from (13) site,these sites were distributed in southern part where sources of pollution found (as ElBatts , El-Wadi drain and the hydraulic pumps ), center of Lake Quaron and the northern part which away from direct sources of pollution as shown in photo (1), these samples were collected during summer and spring seasons (lowest and highest water level) from the study area according to $\boldsymbol{A . P . H . A .}$ (1985).

One liter of water was preconcentrated using APDC and MIBK extraction procedure according to (Brewer et al, 1969; $A P H A, 1985)$ and the results expressed by $\mu \mathrm{g} / \mathrm{l}$. Sediment samples were dried at room temperature then preserved in plastic bags. About $2 \mathrm{~g}$ of dry sediments were heated in water bath at $90^{\circ} \mathrm{C}$ with $4 \mathrm{ml} \mathrm{HNO}_{3}$ and $2 \mathrm{ml}$ $\mathrm{H}_{2} \mathrm{SO}_{4}$ for one hour then allowed to cool, the volume adjusted to $25 \mathrm{ml}$ using deionized water (Adeloju et $\boldsymbol{a l}$, 1994). The results expressed by $\mu \mathrm{g} / \mathrm{g}$ and interpreted with Sediment Quality Guidelines (SQG) according to Persaud et al (1990). Fish samples (10 Sand smelt (Atherina sp.), 10 Bolti (Tilapia zillii), 10 Bouri (Mugil cephalus) and 10 Mousa (Solea vulgaris) of 
variable sizes and weights were collected by fishermen from the study area. Each sample labeled and preserved in icebox then transferred to the laboratory for analysis. (5) g. of fish muscles and the whole fish of Atherina sp were digested by using Nitric/ Percholoric acid (4:1) according to Al Ghais (1995). The results were expressed by $\mu \mathrm{g} / \mathrm{g}$. $\mathrm{Cd}, \mathrm{Cu}, \mathrm{Fe}, \mathrm{Mn}, \mathrm{Ni}, \mathrm{Pb}$ and $\mathrm{Zn}$ were determined using Atomic
Absorption Spectrophotometer (Perkin Elmer, Aanalyst 100 Spectrometer) at National Institute of Oceanography and Fisheries, Suez Branch.

Data were analyzed using SPSS software (version 17.0) according to Snedecor and Cochran (1989). The values were taken as significant at $P$ $\leq 0.05$ ANOVA (analysis of variance).

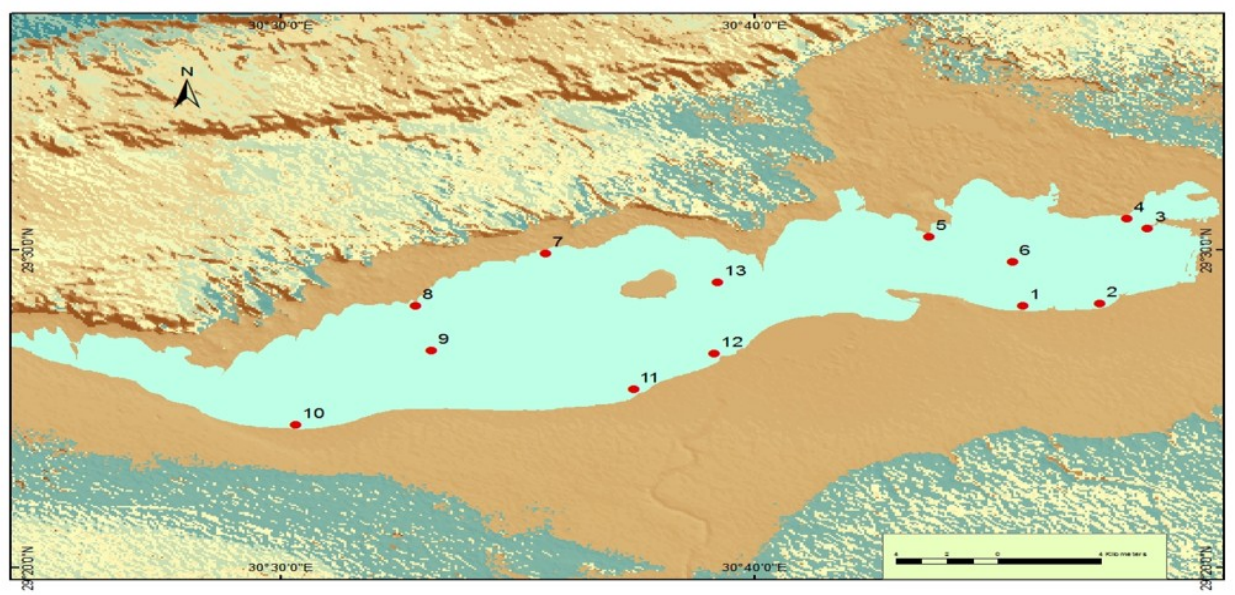

Photo (1): Location of water and sediment sampling sites in Lake Quaron.

\section{RESULTS}

\section{1-Water}

The annual average concentrations $(\mu \mathrm{g} / \mathrm{l})$ of different metals in water samples that were collected from (13) sites from Lake Quaron are presented in

Tables $(1,2)$ and in Figures $(1,2,3,4)$ as follow:-

Site (1) was the highest in different metals except site (3) was higher in $\mathrm{Fe}$ and site (13) was higher in $\mathrm{Cu}$.
The average concentration values of metals (min. -max.) were found as follow; $\mathrm{Cd}$ (0.51-0.144 $\mu \mathrm{g} / \mathrm{l}), \mathrm{Cu}$ $(0.711-1.275 \mu \mathrm{g} / 1)$, Fe (5.361$11.175 \mu \mathrm{g} / \mathrm{l}), \operatorname{Mn}(0.201-2.205 \mu \mathrm{g} / \mathrm{l})$, Ni (0.255-6.39 $\mu \mathrm{g} / \mathrm{l}), \mathrm{Pb}$ (0.333$1.131 \mu \mathrm{g} / \mathrm{l})$ and in $\mathrm{Zn}(1.203-44.736$ $\mu \mathrm{g} / 1)$.

The annual average concentrations of the heavy metals in water samples found to have the following order; $\mathrm{Zn}>\mathrm{Fe}>\mathrm{Ni}>\mathrm{Mn}>\mathrm{Cu}>\mathrm{Pb}>$ Cd. 


\section{2-Sediment}

The annual average concentrations $(\mu \mathrm{g} / \mathrm{g})$ of different metals in water samples that were collected from (13) sites from Lake Quaron are presented in Tables $(3,4)$ and in Figures $(5,6,7,8)$ as follow:-

The average concentration values of metals (min. -max.)Were found as follow; $\mathrm{Cd}$ (0.055-0.94), $\mathrm{Cu}$ (2.13230.27), $\mathrm{Fe}$ (229.57-320.89), $\mathrm{Mn}(56.495-192.18), \quad \mathrm{Ni} \quad(1.59-$ 18.49), $\mathrm{Pb}(1.865-13.5)$ and in $\mathrm{Zn}$ (6.66-36.75).

Site (3) was the highest in different metals levels except site (2) was higher in Mn, site (5) was higher in $\mathrm{Ni}$ and site (6) was higher in $\mathrm{Pb}$.

\section{3-Fish}

The concentration average $(\mu \mathrm{g} / \mathrm{g})$ of $\mathrm{Cd}, \mathrm{Cu}, \mathrm{Fe}, \mathrm{Mn}, \mathrm{Ni}, \mathrm{Pb}$ and $\mathrm{Zn}$ in the muscle tissues of different fish and whole Atherina sp. of Lake Quaron were summarized in Table (5) and illustrated in Figures $(9,10,11,12)$ which were found in the following order :- in Atherina $s p$.as $0.622,4.023,151.49,40.299$, $3.709, \quad 8.885, \quad 28.647 \quad \mu \mathrm{g} / \mathrm{g}$ respectively, in Mugil cephalusas $0.279,2.816,99.399,2.256,2.148$, $2.591,14.266 \mu \mathrm{g} / \mathrm{g}$ respectively, in
Solea vulgaris as $0.224,1.951$, 109.37, 3.262, 1.218, 2.187, 13.674 $\mu \mathrm{g} / \mathrm{g}$ respectively and in Tilapia zillii as $0.355,2.471,117.57,2.086$, $2.517, \quad 3.046, \quad 20.256 \quad \mu \mathrm{g} / \mathrm{g}$ respectively.

The average concentration of metals in different fishes found to have the following order: Atherina sp.>Tilapia zillii $>$ Mugil cephalus $>$ Solea vulgaris except in $\mathrm{Cu}$, Mugil cephalus was higher than tilapia zilli, in $\mathrm{Fe}$ Solea vulgaris higher than Mugil cephalus and in Mn were Atherina sp.>Solea vulgaris $>$ Mugil cephalus $>$ Tilapia zillii.

Statistically table (7) showed that $\mathrm{Cd}, \mathrm{Cu}, \mathrm{Fe}, \mathrm{Mn}, \mathrm{Ni}, \mathrm{Pb}$ and $\mathrm{Zn}$ contents in water were significantly $(\mathrm{P} \leq 0.01)$ and positively correlated with $\mathrm{Cd}, \mathrm{Cu}$, $\mathrm{Fe}, \mathrm{Mn}, \mathrm{Ni}, \mathrm{Pb}$ and $\mathrm{Zn}$ contents respectively in sediment and fish spp.

Table (8) showed that $\mathrm{Cd}, \mathrm{Cu}, \mathrm{Fe}$, $\mathrm{Mn}, \mathrm{Ni}, \mathrm{Pb}$ and $\mathrm{Zn}$ content in fishes were significantly influenced $(\mathrm{P} \leq$ 0.05 ) by species.

Table (9) showed that $\mathrm{Cd}, \mathrm{Cu}, \mathrm{Fe}$, $\mathrm{Mn}, \mathrm{Ni}, \mathrm{Pb}$ and $\mathrm{Zn}$ contents in water and sediment were significantly influenced $(\mathrm{P} \leq 0.05)$ by season. 
Table (1): Heavy metals annual concentration $(\mu \mathrm{g} / \mathrm{l})$ in water collected from Lake Quaron during spring and $\quad$ summer (2013) (average $\pm s d$ ):

\begin{tabular}{|c|c|c|c|c|c|c|c|}
\hline $\begin{array}{c}\text { Annual } \\
\text { Water }\end{array}$ & $\mathbf{C d}$ & $\mathbf{C u}$ & $\mathbf{F e}$ & $\mathbf{M n}$ & $\mathbf{N i}$ & $\mathbf{P b}$ & $\mathbf{Z n}$ \\
\hline \multirow{2}{*}{ Site1 } & $0.144 \pm$ & $0.951 \pm$ & $8.322 \pm$ & $2.205 \pm$ & $6.39 \pm$ & $1.131 \pm$ & $44.736 \pm$ \\
& 0.127 & 0.318 & 2.257 & 2.652 & 8.935 & 1.184 & 59.685 \\
\hline \multirow{2}{*}{ Site2 } & $0.072 \pm$ & $0.888 \pm$ & $7.503 \pm$ & $0.3 \pm 0.0$ & $0.354 \pm$ & $0.621 \pm 0$ & $3.459 \pm 2$. \\
& 0.008 & 0.034 & 1.549 & 59 & 0.161 & .148 & 796 \\
\hline \multirow{2}{*}{ Site3 } & $0.084 \pm$ & $1.008 \pm$ & 11.175 & $0.486 \pm$ & $0.3 \pm 0.1$ & $0.549 \pm 0$ & $4.941 \pm 1$. \\
& 0.008 & 0.042 & \pm 1.532 & 0.255 & 02 & .055 & 286 \\
\hline \multirow{2}{*}{ Site4 } & $0.096 \pm$ & $0.774 \pm$ & $6.648 \pm$ & $0.303 \pm$ & $0.255 \pm$ & $0.417 \pm 0$ & $1.662 \pm 0$. \\
& 0.008 & 0.093 & 0.365 & 0.064 & 0.208 & .038 & 959 \\
\hline \multirow{2}{*}{ Site5 } & $0.09 \pm$ & $0.738 \pm$ & $5.403 \pm$ & $0.279 \pm$ & $0.471 \pm$ & $0.384 \pm 0$ & $1.203 \pm 0$. \\
& 0.008 & 0.025 & 0.938 & 0.106 & 0.250 & .093 & 411 \\
\hline \multirow{2}{*}{ Site6 } & $0.075 \pm$ & $0.711 \pm$ & $6.459 \pm$ & $0.285 \pm$ & $0.528 \pm$ & $0.339 \pm 0$ & $2.1 \pm 1.57$ \\
& 0.021 & 0.064 & 0.242 & 0.039 & 0.187 & .115 & 9 \\
\hline \multirow{2}{*}{ Site7 } & $0.057 \pm$ & $0.879 \pm$ & $7.368 \pm$ & $0.318 \pm$ & $0.462 \pm$ & $0.333 \pm 0$ & $6.996 \pm 1$. \\
& 0.004 & 0.055 & 2.613 & 0.0 & 0.475 & .098 & 782 \\
\hline \multirow{2}{*}{ Site8 } & $0.051 \pm$ & $0.903 \pm$ & $6.399 \pm$ & $0.201 \pm$ & $0.45 \pm 0$. & $0.414 \pm 0$ & $5.229 \pm 6$. \\
& 0.013 & 0.064 & 0.148 & 0.013 & 305 & .187 & 105 \\
\hline \multirow{2}{*}{ Site9 } & $0.06 \pm$ & $0.888 \pm$ & $5.361 \pm$ & $0.252 \pm$ & $0.441 \pm$ & $0.465 \pm 0$ & $1.653 \pm 0$. \\
& 00 & 0.297 & 0.386 & 0.043 & 0.089 & .386 & 199 \\
\hline \multirow{2}{*}{ Site10 } & $0.085 \pm$ & $0.972 \pm$ & $6.981 \pm$ & $0.312 \pm$ & $0.484 \pm$ & $0.647 \pm 0$ & $1.316 \pm 0$. \\
& 0.037 & 0.271 & 2.188 & 0.110 & 0.262 & .106 & 602 \\
\hline \multirow{2}{*}{ Site11 } & $0.063 \pm$ & $0.969 \pm$ & $6.858 \pm$ & $0.243 \pm$ & $0.42 \pm 0$. & $0.768 \pm 0$ & $1.695 \pm 1$. \\
& 0.013 & 0.047 & 0.229 & 0.021 & 433 & .179 & 201 \\
\hline \multirow{2}{*}{ Site12 } & $0.078 \pm$ & $0.918 \pm$ & $6.375 \pm$ & $0.3 \pm 0.0$ & $0.267 \pm$ & $0.57 \pm 0$. & $1.827 \pm 1$. \\
& 0.008 & 0.008 & 0.301 & 08 & 0.165 & 178 & 439 \\
\hline \multirow{2}{*}{ Site13 } & $0.092 \pm$ & $1.275 \pm$ & 11.029 & $0.228 \pm$ & $0.476 \pm$ & $0.341 \pm 0$ & $3.272 \pm 2$. \\
& 0.014 & 0.505 & \pm 0.529 & 0.025 & 0.039 & .078 & 047 \\
\hline
\end{tabular}

Table (2): Comparison of heavy metals concentration $(\mu \mathrm{g} / L)$ in water of Lake Quaron (2013) with previous studies.

\begin{tabular}{|l|l|l|l|l|l|l|l|l|}
\hline Area-water & $\mathbf{C d}$ & $\mathbf{C u}$ & $\mathbf{F e}$ & $\mathbf{M n}$ & $\mathbf{N i}$ & $\mathbf{P b}$ & \multicolumn{1}{|c|}{$\mathbf{Z n}$} & References \\
\hline $\begin{array}{l}\text { Lake } \\
\text { Quaron }\end{array}$ & $\begin{array}{l}0.051 \\
-\end{array}$ & $\begin{array}{l}0.711- \\
1.275\end{array}$ & $\begin{array}{l}5.361- \\
11.17\end{array}$ & $\begin{array}{l}0.201- \\
2.205\end{array}$ & $\begin{array}{l}0.255- \\
6.39\end{array}$ & $\begin{array}{l}0.333- \\
1.131\end{array}$ & $\begin{array}{l}1.203 \\
- \\
44.736\end{array}$ & Present study \\
\hline $\begin{array}{l}\text { Lake } \\
\text { Quaron }\end{array}$ & $\begin{array}{l}1.58- \\
2.21\end{array}$ & $\begin{array}{l}35.80- \\
56.50\end{array}$ & $\begin{array}{l}0.33- \\
0.60\end{array}$ & $\begin{array}{l}39.10- \\
78.30\end{array}$ & $\begin{array}{l}38.81 \\
-44.0\end{array}$ & $\begin{array}{l}84.50 \\
- \\
99.85\end{array}$ & $\begin{array}{l}28.50 \\
-\end{array}$ & $\begin{array}{l}\text { Ali and Fishar } \\
\text { (2005) }\end{array}$ \\
\hline $\begin{array}{l}\text { Lake } \\
\text { Quaron }\end{array}$ & - & 40 & 346 & 51 & - & 384 & 626 & $\begin{array}{l}\text { Mohamed and } \\
\text { Gad (2008) }\end{array}$ \\
\hline $\begin{array}{l}\text { Lake } \\
\text { Quaron }\end{array}$ & $\begin{array}{l}5.0- \\
77.33\end{array}$ & $\begin{array}{l}21.0- \\
56.50\end{array}$ & $\begin{array}{l}434.2 \\
7.33 .0\end{array}$ & $\begin{array}{l}31.0- \\
131.83\end{array}$ & - & $\begin{array}{l}38.0- \\
400.8\end{array}$ & $\begin{array}{l}64.0-85.33 \\
8\end{array}$ & $\begin{array}{l}\text { Abdel-Satar et } \\
\text { al.(2010) }\end{array}$ \\
\hline $\begin{array}{l}\text { Lake } \\
\text { Quaron }\end{array}$ & 96.8 & 96.9 & 625.6 & 111.8 & - & 106.0 & 85.0 & $\begin{array}{l}\text { Ibrahim and } \\
\text { Ramzy(2013) }\end{array}$ \\
\hline
\end{tabular}




\begin{tabular}{|l|l|l|l|l|l|l|l|l|}
\hline $\begin{array}{l}\text { Lake } \\
\text { Borollus }\end{array}$ & 7.06 & 12.68 & 19.14 & & & 7.27 & 15.56 & $\begin{array}{l}\text { Shakweer and } \\
\text { Radwan (2004) }\end{array}$ \\
\hline Lake Edku & $\begin{array}{l}11.25- \\
11.75\end{array}$ & $\begin{array}{l}20- \\
31.5\end{array}$ & $\begin{array}{l}300- \\
820\end{array}$ & $\begin{array}{l}28.83- \\
75.25\end{array}$ & $\begin{array}{l}26.25- \\
51.50\end{array}$ & $\begin{array}{l}18.5- \\
95.5\end{array}$ & $\begin{array}{l}8.85- \\
57.55\end{array}$ & $\begin{array}{l}\text { Masoud et al. } \\
\text { (2005) }\end{array}$ \\
\hline $\begin{array}{l}\text { Lake } \\
\text { Manzala }\end{array}$ & 20.0 & 55.0 & - & - & - & 22.0 & 311 & $\begin{array}{l}\text { Bahnasawy et } \\
\text { al., (2011) }\end{array}$ \\
\hline $\begin{array}{l}\text { Lake } \\
\text { Manzala }\end{array}$ & 92 & 1351 & - & - & - & 1320 & 3363 & $\begin{array}{l}\text { Salah-Eldein } \\
\text { (2012) }\end{array}$ \\
\hline $\begin{array}{l}\text { Permissible } \\
\text { limit }\end{array}$ & 10 & 1000 & 300 & 1000 & 632 & 50 & 5000 & USEPA (1986) \\
\hline
\end{tabular}

Table (3): Heavy metals annual concentration $(\mu \mathrm{g} / l)$ in sediment collected from Lake Quaron during (2013) (average $\pm s d$ ):

\begin{tabular}{|c|c|c|c|c|c|c|c|}
\hline$\approx \varnothing:$ & Cd & $\mathbf{C u}$ & $\mathrm{Fe}$ & Mn & $\mathbf{N i}$ & $\mathbf{P b}$ & $\mathbf{Z n}$ \\
\hline Site1 & $\begin{array}{c}0.94 \pm 0.05 \\
7\end{array}$ & $10.145 \pm 2.864$ & $270.6 \pm 2.758$ & 2 & $\begin{array}{c}2.565 \pm 1.59 \\
1\end{array}$ & $\begin{array}{c}12.345 \pm 1.8 \\
88\end{array}$ & $\begin{array}{c}14.795 \pm 1.8 \\
46\end{array}$ \\
\hline Site2 & $\begin{array}{c}0.585 \pm 0.0 \\
21\end{array}$ & $17.92 \pm 16.292$ & $\begin{array}{c}264.678 \pm 2.2 \\
34\end{array}$ & $\begin{array}{c}192.178 \pm 41 \\
123\end{array}$ & $\begin{array}{c}9.325 \pm 11.7 \\
59\end{array}$ & $\begin{array}{c}10.63 \pm 1.28 \\
7\end{array}$ & $\begin{array}{c}14.405 \pm 5.6 \\
78\end{array}$ \\
\hline Site3 & $\begin{array}{c}0.619 \pm 0.0 \\
44\end{array}$ & $\begin{array}{c}230.265 \pm 275 \\
977\end{array}$ & $\begin{array}{c}320.797 \pm 64 \\
696\end{array}$ & $\begin{array}{c}138.192 \pm 6.3 \\
61\end{array}$ & $\begin{array}{c}13.471 \pm 0.6 \\
94\end{array}$ & $\begin{array}{c}11.783 \pm 1.7 \\
49\end{array}$ & $\begin{array}{c}36.747 \pm 16 . \\
386\end{array}$ \\
\hline Site4 & $\begin{array}{c}0.364 \pm 0.3 \\
59\end{array}$ & $\begin{array}{c}157.551 \pm 219 \\
501\end{array}$ & $\begin{array}{c}285.125 \pm 75 \\
483\end{array}$ & $\begin{array}{c}56.495 \pm 54.1 \\
43\end{array}$ & $\begin{array}{c}11.787 \pm 4.8 \\
74\end{array}$ & $\begin{array}{c}6.739 \pm 7.35 \\
3\end{array}$ & $\begin{array}{c}24.523 \pm 19 . \\
337\end{array}$ \\
\hline Site5 & $\begin{array}{c}0.235 \pm 0.0 \\
92\end{array}$ & $5.165 \pm$ & $\begin{array}{c}256.248 \pm 0.5 \\
27\end{array}$ & $\begin{array}{c}97.245 \pm 45.6 \\
86\end{array}$ & $\begin{array}{c}18.49 \pm 7.89 \\
1\end{array}$ & $3.9 \pm 0.806$ & $\begin{array}{c}10.025 \pm 1.2 \\
09\end{array}$ \\
\hline Site6 & $\begin{array}{c}0.62 \pm 0.01 \\
4\end{array}$ & $41.42 \pm 29.571$ & $\begin{array}{c}272.475 \pm 0.2 \\
33\end{array}$ & $\begin{array}{c}152.699 \pm 3.2 \\
41\end{array}$ & $4.67 \pm 4.158$ & $13.5 \pm 2.121$ & $\begin{array}{c}28.925 \pm 13 . \\
315\end{array}$ \\
\hline Site7 & $\begin{array}{c}0.055 \pm 0.0 \\
64\end{array}$ & $2.295 \pm 0.389$ & $\begin{array}{c}235.766 \pm 7.6 \\
71\end{array}$ & $\begin{array}{c}90.923 \pm 95.5 \\
49\end{array}$ & $\begin{array}{c}2.385 \pm 1.50 \\
6\end{array}$ & $1.97 \pm 0.523$ & $6.66 \pm 0.608$ \\
\hline Site8 & $\begin{array}{c}0.11 \pm 0.07 \\
1\end{array}$ & $2.13 \pm 0.099$ & $\begin{array}{c}229.565 \pm 1.8 \\
88\end{array}$ & $\begin{array}{c}98.332 \pm 84.6 \\
99\end{array}$ & $\begin{array}{c}15.97 \pm 6.50 \\
5\end{array}$ & $\begin{array}{c}1.865 \pm 0.56 \\
8\end{array}$ & $\begin{array}{c}6.745 \pm 1.13 \\
8\end{array}$ \\
\hline Site9 & $\begin{array}{c}0.52 \pm 0.14 \\
1\end{array}$ & $20.22 \pm 0.481$ & $\begin{array}{c}272.305 \pm 0.5 \\
02\end{array}$ & $\begin{array}{c}100.845 \pm 59 \\
630\end{array}$ & $1.59 \pm 0.240$ & $\begin{array}{c}11.37 \pm 0.32 \\
5\end{array}$ & $\begin{array}{c}28.53 \pm 15.1 \\
32\end{array}$ \\
\hline $\begin{array}{c}\text { Site } 1 \\
0\end{array}$ & $\begin{array}{c}0.63 \pm 0.05 \\
7\end{array}$ & $8.545 \pm 0.879$ & $\begin{array}{c}268.015 \pm 0.8 \\
79\end{array}$ & $\begin{array}{c}104.545 \pm 66 \\
956\end{array}$ & $7.59 \pm 8.796$ & $\begin{array}{c}9.325 \pm 0.94 \\
0\end{array}$ & $\begin{array}{c}13.36 \pm 3.32 \\
3\end{array}$ \\
\hline $\begin{array}{c}\text { Site1 } \\
1\end{array}$ & $\begin{array}{c}0.595 \pm 0.0 \\
78\end{array}$ & $12.08 \pm 7.396$ & $\begin{array}{c}268.085 \pm 2.7 \\
93\end{array}$ & $\begin{array}{c}154.099 \pm 13 \\
108\end{array}$ & $\begin{array}{c}16.59 \pm 4.93 \\
6\end{array}$ & $9.63 \pm 00$ & $\begin{array}{c}14.755 \pm 3.5 \\
99\end{array}$ \\
\hline $\begin{array}{l}\text { Site } 1 \\
2\end{array}$ & $\begin{array}{c}0.51 \pm 0.12 \\
7\end{array}$ & $12.565 \pm 3.387$ & $\begin{array}{c}269.385 \pm 1.8 \\
69\end{array}$ & $\begin{array}{c}106.18 \pm 54.1 \\
64\end{array}$ & $6.31 \pm 5.473$ & $\begin{array}{c}10.33 \pm 0.39 \\
6\end{array}$ & $\begin{array}{c}24.125 \pm 10 . \\
034\end{array}$ \\
\hline $\begin{array}{l}\text { Site } 1 \\
3\end{array}$ & $\begin{array}{c}0.525 \pm 0.0 \\
78\end{array}$ & $4.27 \pm 1.499$ & $\begin{array}{c}252.92 \pm 7.34 \\
9\end{array}$ & $\begin{array}{c}129.895 \pm 23 \\
879\end{array}$ & $\begin{array}{c}16.93 \pm 10.7 \\
62\end{array}$ & $5.84 \pm 1.895$ & $\begin{array}{c}21.7 \pm 19.79 \\
9\end{array}$ \\
\hline
\end{tabular}


Table (4): Comparison of heavy metals concentration ( $\mu \mathrm{g} / \mathrm{g})$ in sediment of Lake Quaron(2013) with previous studies.

\begin{tabular}{|c|c|c|c|c|c|c|c|c|}
\hline Area-sed. & Cd & $\mathrm{Cu}$ & $\mathrm{Fe}$ & Mn & $\mathbf{N i}$ & $\mathbf{P b}$ & $\mathbf{Z n}$ & References \\
\hline $\begin{array}{c}\text { Lake } \\
\text { Quaron }\end{array}$ & $\begin{array}{c}0.055- \\
0.94\end{array}$ & $\begin{array}{c}2.13- \\
230.27\end{array}$ & $\begin{array}{l}229.57- \\
320.89\end{array}$ & $\begin{array}{c}56.495- \\
192.18 \\
\end{array}$ & $\begin{array}{l}1.59- \\
18.49\end{array}$ & $\begin{array}{c}1.865- \\
13.5\end{array}$ & $\begin{array}{l}6.66- \\
36.75\end{array}$ & $\begin{array}{c}\text { Present } \\
\text { study }\end{array}$ \\
\hline $\begin{array}{l}\text { Lake } \\
\text { Quaron }\end{array}$ & $\begin{array}{c}0.97- \\
1.80\end{array}$ & $\begin{array}{c}23.76- \\
54.50\end{array}$ & $\begin{array}{c}11.19- \\
26.38\end{array}$ & $\begin{array}{c}213.58- \\
456.30\end{array}$ & $\begin{array}{c}38.78- \\
83.60\end{array}$ & $\begin{array}{c}16.98- \\
24.8\end{array}$ & $\begin{array}{l}72.50- \\
180.49\end{array}$ & $\begin{array}{l}\text { Ali and } \\
\text { Fishar } \\
(2005) \\
\end{array}$ \\
\hline $\begin{array}{c}\text { Lake } \\
\text { Quaron }\end{array}$ & $\begin{array}{l}5.80- \\
11.50\end{array}$ & $\begin{array}{l}38.8- \\
85.50\end{array}$ & $\begin{array}{c}2.590- \\
2.730\end{array}$ & $\begin{array}{c}492.30- \\
1097.8\end{array}$ & - & $\begin{array}{l}37.0- \\
76.80\end{array}$ & $\begin{array}{c}115.80- \\
190.30\end{array}$ & $\begin{array}{c}\text { Abdel- } \\
\text { Satar et al. } \\
(2010)\end{array}$ \\
\hline $\begin{array}{l}\text { Lake } \\
\text { Edku }\end{array}$ & $\begin{array}{l}0.62- \\
1.56\end{array}$ & $\begin{array}{c}11.83- \\
2.57\end{array}$ & $\begin{array}{l}27400- \\
36290\end{array}$ & $\begin{array}{l}226.35- \\
273.17\end{array}$ & $\begin{array}{l}13.79- \\
18.35\end{array}$ & $\begin{array}{l}1.15- \\
2.41\end{array}$ & $52-71.25$ & $\begin{array}{c}\text { Masoud et } \\
\text { al. } \\
(2005) \\
\end{array}$ \\
\hline $\begin{array}{c}\text { Lake } \\
\text { Manzala }\end{array}$ & 1.567 & 21.065 & - & - & - & 10.243 & 173.108 & $\begin{array}{l}\text { Salah- } \\
\text { Eldein } \\
\text { (2012) }\end{array}$ \\
\hline $\begin{array}{c}{ }^{*} \text { SQG } \\
(\mu \mathrm{g} / \mathrm{g} \text { dry } \\
\text { wt) }\end{array}$ & $\begin{array}{c}0.6- \\
10.0\end{array}$ & $\begin{array}{l}16- \\
110\end{array}$ & $\begin{array}{c}2 \%- \\
4 \%\end{array}$ & $\begin{array}{l}460- \\
1100\end{array}$ & $16-75$ & $\begin{array}{l}31- \\
250\end{array}$ & $\begin{array}{l}120- \\
820\end{array}$ & $\begin{array}{l}\text { Persaud et } \\
\text { al. (1990) }\end{array}$ \\
\hline
\end{tabular}

*Sediment quality guidelines: it showed two levels: The lowest level indicates a level of contamination which has no effect on the majority of the sediment-dwelling organisms while the highest level representing the sediment is considered heavily polluted and likely to affect the health of sediment-dwelling organisms, at the Severe Effect Level a management plan may be required.

Table (5): Heavy metals concentration ( $\mu \mathrm{g} / \mathrm{g}$ dry wt.) in fishes collected from during Lake Quaron(2013):

\begin{tabular}{|c|c|c|c|c|c|c|c|}
\hline Species & Cd & $\mathrm{Cu}$ & $\mathbf{F e}$ & Mn & $\mathbf{N i}$ & $\mathbf{P b}$ & $\mathbf{Z n}$ \\
\hline Atherinasp & $\begin{array}{c}0.622 \pm 0.1 \\
84\end{array}$ & $\begin{array}{c}4.0231 \pm 0.6 \\
73\end{array}$ & $\begin{array}{c}151.399 \pm 50 \\
801\end{array}$ & $\begin{array}{c}40.299 \pm 17 \\
443\end{array}$ & $\begin{array}{c}3.709 \pm 1.1 \\
93\end{array}$ & $\begin{array}{c}8.885 \pm 2.6 \\
39\end{array}$ & $\begin{array}{c}28.647 \pm 7.6 \\
43\end{array}$ \\
\hline $\begin{array}{c}\text { Mugilcepha } \\
\text { lus }\end{array}$ & $\begin{array}{c}0.279 \pm 0.0 \\
54\end{array}$ & $\begin{array}{c}2.816 \pm 0.59 \\
5\end{array}$ & $\begin{array}{c}99.399 \pm 15.3 \\
61\end{array}$ & $\begin{array}{c}2.256 \pm 0.47 \\
7\end{array}$ & $\begin{array}{c}2.148 \pm 0.6 \\
71\end{array}$ & $\begin{array}{c}2.591 \pm 0.6 \\
29\end{array}$ & $\begin{array}{c}14.266 \pm 2.3 \\
94\end{array}$ \\
\hline $\begin{array}{c}\text { Solea } \\
\text { vulgaris }\end{array}$ & $\begin{array}{c}0.224 \pm 0.1 \\
32\end{array}$ & $\begin{array}{c}1.951 \pm 0.57 \\
4\end{array}$ & $\begin{array}{c}109.367 \pm 23 \\
807\end{array}$ & $\begin{array}{c}3.262 \pm 1.32 \\
2\end{array}$ & $\begin{array}{c}1.218 \pm 0.3 \\
30\end{array}$ & $\begin{array}{c}2.187 \pm 0.4 \\
91\end{array}$ & $\begin{array}{c}13.674 \pm 1.8 \\
75\end{array}$ \\
\hline Tilapia zilli & $\begin{array}{c}0.355 \pm 0.0 \\
64\end{array}$ & $\begin{array}{c}2.471 \pm 0.48 \\
8\end{array}$ & $\begin{array}{c}117.57 \pm 37.7 \\
08\end{array}$ & $\begin{array}{c}2.086 \pm 0.42 \\
0\end{array}$ & $\begin{array}{c}2.517 \pm 0.4 \\
91\end{array}$ & $\begin{array}{c}3.046 \pm 0.8 \\
99\end{array}$ & $\begin{array}{c}20.256 \pm 4.1 \\
71\end{array}$ \\
\hline
\end{tabular}


Table (6): Comparison of heavy metals concentration ( $\mu \mathrm{g} / \mathrm{g} d r y$ wt.) in fish muscles of Lake Quaron(2013) with previous studies.

\begin{tabular}{|c|c|c|c|c|c|c|c|c|c|}
\hline Area & Species & Cd & $\mathrm{Cu}$ & $\mathbf{F e}$ & Mn & $\mathbf{N i}$ & $\mathbf{P b}$ & Zn & References \\
\hline \multirow{4}{*}{$\begin{array}{l}\text { Lake } \\
\text { Quaron }\end{array}$} & Atherina sp. & 0.622 & 4.023 & 151.49 & 40.299 & 3.709 & 8.885 & 28.647 & \multirow{4}{*}{$\begin{array}{l}\text { Present } \\
\text { study }\end{array}$} \\
\hline & $\begin{array}{l}\text { Mugil } \\
\text { cephalus }\end{array}$ & 0.279 & 2.816 & 99.399 & 2.256 & 2.148 & 2.591 & 14.266 & \\
\hline & $\begin{array}{l}\text { Solea } \\
\text { vulgaris }\end{array}$ & 0.224 & 1.951 & 109.37 & 3.262 & 1.218 & 2.187 & 13.674 & \\
\hline & Tilapia zilli & 0.355 & 2.471 & 117.57 & 2.086 & 2.517 & 3.046 & 20.256 & \\
\hline \multirow{4}{*}{$\begin{array}{l}\text { Lake } \\
\text { Quaron }\end{array}$} & Mugil sp. & $\begin{array}{l}1.04- \\
1.16\end{array}$ & $\begin{array}{l}8.91- \\
9.00\end{array}$ & $\begin{array}{l}55.74- \\
85.56\end{array}$ & $\begin{array}{ll}7.01 & - \\
8.38 & \end{array}$ & $\begin{array}{l}6.04- \\
6.72\end{array}$ & $\begin{array}{l}6.36- \\
6.62\end{array}$ & $\begin{array}{l}27.52 \\
30.84\end{array}$ & \multirow{4}{*}{$\begin{array}{l}\text { Ali and } \\
\text { Fishar } \\
(\mathbf{2 0 0 5 )}\end{array}$} \\
\hline & Solea sp. & $\begin{array}{l}1.58- \\
1.87\end{array}$ & $\begin{array}{l}9.38- \\
13.68\end{array}$ & $\begin{array}{l}43.35- \\
82.35\end{array}$ & $\begin{array}{l}7.20 \quad- \\
12.59\end{array}$ & $\begin{array}{l}5.66- \\
6.23\end{array}$ & $\begin{array}{l}6.34- \\
8.16\end{array}$ & $\begin{array}{ll}29.18 \quad- \\
32.11 & \end{array}$ & \\
\hline & Tilapia sp. & $\begin{array}{l}1.10- \\
1.33\end{array}$ & $\begin{array}{l}6.43- \\
10.54\end{array}$ & $\begin{array}{l}19.61- \\
42.39\end{array}$ & $\begin{array}{ll}5.47 & - \\
8.82 & \end{array}$ & $\begin{array}{l}5.42- \\
5.53\end{array}$ & $\begin{array}{l}5.82- \\
7.86\end{array}$ & $\begin{array}{ll}22.83 & - \\
26.29 & \end{array}$ & \\
\hline & Crustacea & $\begin{array}{l}0.15- \\
0.24\end{array}$ & $\begin{array}{l}5.32- \\
8.16\end{array}$ & $\begin{array}{l}44.39- \\
73.27\end{array}$ & $\begin{array}{l}6.50 \quad- \\
11.04\end{array}$ & $\begin{array}{l}6.02- \\
10.53\end{array}$ & $\begin{array}{l}2.47- \\
3.68\end{array}$ & $\begin{array}{l}20.29 \quad- \\
21.45\end{array}$ & \\
\hline \multirow{2}{*}{$\begin{array}{l}\text { Lake } \\
\text { Quaron }\end{array}$} & $\begin{array}{l}\text { Mugil } \\
\text { cephalus }\end{array}$ & - & $\begin{array}{l}1.31- \\
5.01\end{array}$ & - & - & - & - & $\begin{array}{ll}0.79 & - \\
3.85 & \end{array}$ & \multirow{2}{*}{$\begin{array}{l}\text { Authman } \\
\text { and Abbas } \\
(2007)\end{array}$} \\
\hline & Tilapia zilli & - & $\begin{array}{l}5.93- \\
17.85\end{array}$ & - & - & - & - & $\begin{array}{ll}3.0 & - \\
13.56 & \end{array}$ & \\
\hline \multirow{3}{*}{$\begin{array}{l}\text { Lake } \\
\text { Quaron }\end{array}$} & Mugil capito & - & 1.75 & 46.25 & 4.25 & - & 11.88 & 46.38 & \multirow{3}{*}{$\begin{array}{l}\text { Mohamed } \\
\text { and Gad } \\
(2008)\end{array}$} \\
\hline & $\begin{array}{l}\text { Solea } \\
\text { vulgaris }\end{array}$ & - & 3.88 & 53.67 & 12.63 & - & 8.50 & 60.38 & \\
\hline & Tilapia zilli & - & 1.75 & 42.81 & 10.25 & - & 19.50 & 55.88 & \\
\hline Lake Edku & $\begin{array}{l}\text { Oreochromis } \\
\text { niloticus }\end{array}$ & 0.19 & 2.80 & 75.19 & 1.98 & - & 0.59 & 27.60 & \multirow{2}{*}{$\begin{array}{l}\text { Saeed and } \\
\text { Shaker } \\
(\mathbf{2 0 0 8 )}\end{array}$} \\
\hline $\begin{array}{l}\text { Lake } \\
\text { Borollus }\end{array}$ & $\begin{array}{l}\text { Oreochromis } \\
\text { niloticus }\end{array}$ & 0.014 & 1.77 & 21.44 & 0.23 & - & 0.016 & 9.88 & \\
\hline $\begin{array}{l}\text { Lake } \\
\text { Manzala }\end{array}$ & Tilapia sp. & 0.491 & 2.057 & - & - & - & 2.612 & 160.222 & $\begin{array}{l}\text { Salah-Eldein } \\
(2012)\end{array}$ \\
\hline $\begin{array}{l}\text { Permissible } \\
\text { limits }\end{array}$ & & 1.0 & 30 & 100 & 1.0 & $\begin{array}{l}0.5- \\
0.6\end{array}$ & 2.0 & 100 & $\begin{array}{l}\text { WHO(1989) } \\
\text { FAO (1983). }\end{array}$ \\
\hline
\end{tabular}

Permissible limits (dry wt.) according to WHO (1989), FAO (1983). 
Table (7): Correlation coefficients between heavy metals content (i.e. Cd, $\mathrm{Cu}, \mathrm{Fe}, \mathrm{Mn}, \mathrm{Ni}, \mathrm{Pb}$ and $\mathrm{Zn}$ ) in water, sediment and fishes collected from Quaron Lake during (2013).

\begin{tabular}{|c|c|c|c|c|c|c|c|c|c|c|c|c|}
\hline \multirow[b]{2}{*}{$\begin{array}{l}\text { Sour } \\
\text { ces }\end{array}$} & \multicolumn{6}{|l|}{ Cd } & \multicolumn{6}{|l|}{$\mathrm{Cu}$} \\
\hline & $\begin{array}{l}\text { Wat } \\
\text { er }\end{array}$ & $\begin{array}{l}\text { Sedim } \\
\text { ent }\end{array}$ & $\begin{array}{l}\text { Mugi } \\
\text { I } \\
\text { ceph } \\
\text { alus }\end{array}$ & $\begin{array}{l}\text { Tila } \\
\text { pia } \\
\text { zillii }\end{array}$ & $\begin{array}{l}\text { Solea } \\
\text { vulg } \\
\text { aris }\end{array}$ & $\begin{array}{l}\text { Ather } \\
\text { ina } \\
\text { sp. }\end{array}$ & $\begin{array}{l}\text { Wat } \\
\text { er }\end{array}$ & $\begin{array}{l}\text { Sedim } \\
\text { ent }\end{array}$ & $\begin{array}{l}\text { Mugi } \\
\text { I } \\
\text { ceph } \\
\text { alus }\end{array}$ & $\begin{array}{l}\text { Tila } \\
\text { pia } \\
\text { zillii }\end{array}$ & $\begin{array}{l}\text { Solea } \\
\text { vulg } \\
\text { aris }\end{array}$ & $\begin{array}{l}\text { Ather } \\
\text { ina } \\
\text { sp. }\end{array}$ \\
\hline $\begin{array}{l}\text { Wate } \\
\mathbf{r}\end{array}$ & 1 & & & & & & 1 & & & & & \\
\hline $\begin{array}{l}\text { Sedi } \\
\text { ment }\end{array}$ & $\begin{array}{l}0.92 \\
1^{* *} \\
\end{array}$ & 1 & & & & & $\begin{array}{l}0.94 \\
6^{* *} \\
\end{array}$ & 1 & & & & \\
\hline $\begin{array}{l}\text { Mugil } \\
\text { cepha } \\
\text { lus }\end{array}$ & $\begin{array}{l}0.98 \\
0^{* * *}\end{array}$ & $0.980^{*}$ & 1 & & & & $\begin{array}{l}0.99 \\
6^{* *}\end{array}$ & 0.914 & 1 & & & \\
\hline $\begin{array}{l}\text { Tilapi } \\
\text { a zillii }\end{array}$ & $\begin{array}{l}0.96 \\
6^{* *} \\
\end{array}$ & $0.990^{*}$ & 0.998 & 1 & & & $\begin{array}{l}0.98 \\
5^{* *} \\
\end{array}$ & $0.875^{*}$ & 0.996 & 1 & & \\
\hline $\begin{array}{l}\text { Solea } \\
\text { vulga } \\
\text { ris } \\
\end{array}$ & $\begin{array}{l}0.99 \\
0^{* * *} \\
\end{array}$ & $0.967^{*}$ & $\underset{* *}{0.998}$ & $\begin{array}{l}0.99 \\
3^{* *}\end{array}$ & 1 & & $\begin{array}{l}0.99 \\
0^{* *}\end{array}$ & $0.982^{*}$ & 0.973 & $\begin{array}{l}0.95 \\
0^{* *}\end{array}$ & 1 & \\
\hline $\begin{array}{l}\text { Ather } \\
\text { ina } \\
\text { sp. } \\
\end{array}$ & $\begin{array}{l}0.99 \\
3^{* *} \\
\end{array}$ & $0.961^{*}$ & $\underset{* *}{0.997}$ & $\begin{array}{l}0.99 \\
0^{* *} \\
\end{array}$ & 1.000 & 1 & $\begin{array}{l}0.98 \\
3^{* *} \\
\end{array}$ & ${ }_{*}^{0.871^{*}}$ & 0.996 & $\begin{array}{l}1.00 \\
0^{* *} \\
\end{array}$ & $\underset{* *}{0.947}$ & 1 \\
\hline $\begin{array}{l}\text { Sourc } \\
\text { es }\end{array}$ & \multicolumn{6}{|l|}{$\mathrm{Fe}$} & \multicolumn{6}{|l|}{ Mn } \\
\hline $\begin{array}{l}\text { Wate } \\
\mathbf{r}\end{array}$ & 1 & & & & & & 1 & & & & & \\
\hline $\begin{array}{l}\text { Sedi } \\
\text { ment }\end{array}$ & $\begin{array}{l}0.98 \\
0^{* *}\end{array}$ & 1 & & & & & $\begin{array}{l}0.94 \\
7^{\text {** }}\end{array}$ & 1 & & & & \\
\hline $\begin{array}{l}\text { Mugil } \\
\text { cepha } \\
\text { lus } \\
\end{array}$ & $\begin{array}{l}0.98 \\
8^{* * *} \\
\end{array}$ & $0.999^{*}$ & 1 & & & & $\begin{array}{l}0.90 \\
2^{* *}\end{array}$ & $0.993^{*}$ & 1 & & & \\
\hline $\begin{array}{l}\text { Tilapi } \\
\text { a zillii }\end{array}$ & $\begin{array}{l}1.00 \\
0^{* * *}\end{array}$ & $0.979^{*}$ & 0.987 & 1 & & & $\begin{array}{l}0.88 \\
9^{* *}\end{array}$ & $0.989^{*}$ & 1.000 & 1 & & \\
\hline $\begin{array}{l}\text { Solea } \\
\text { vulga } \\
\text { ris }\end{array}$ & $\begin{array}{l}0.99 \\
1^{* *}\end{array}$ & $0.998^{*}$ & 1.000 & $\begin{array}{l}\mathbf{0} \\
.990 \\
* *\end{array}$ & 1 & & $\begin{array}{l}0.79 \\
4^{*}\end{array}$ & $0.947^{*}$ & $\underset{* *}{0.979}$ & $\begin{array}{l}0.98 \\
4^{* * *}\end{array}$ & 1 & \\
\hline \multirow[t]{2}{*}{$\begin{array}{l}\text { Ather } \\
\text { ina } \\
\text { sp. } \\
\end{array}$} & $\underset{6.96}{0.96}$ & $0.897^{*}$ & $\underset{* *}{0.916}$ & $\begin{array}{l}0.96 \\
8^{* *} \\
\end{array}$ & $\underset{* * *}{0.924}$ & 1 & $\begin{array}{l}0.90 \\
6^{* *}\end{array}$ & $0.994^{*}$ & $\underset{* *}{1.000}$ & $\begin{array}{l}0.99 \\
9^{* * *}\end{array}$ & $\underset{* * *}{\mathbf{0 . 9 7 7}}$ & 1 \\
\hline & \multicolumn{6}{|l|}{\begin{tabular}{|l}
$\mathrm{Ni}$ \\
\end{tabular}} & \multicolumn{6}{|l|}{$\mathbf{P b}$} \\
\hline $\begin{array}{l}\text { Wate } \\
\mathbf{r}\end{array}$ & 1 & & & & & & 1 & & & & & \\
\hline $\begin{array}{l}\text { Sedi } \\
\text { ment }\end{array}$ & $\begin{array}{l}0.85 \\
9^{* * *} \\
\end{array}$ & 1 & & & & & $\begin{array}{l}0.92 \\
8^{* *}\end{array}$ & 1 & & & & \\
\hline $\begin{array}{l}\text { Mugil } \\
\text { cepha } \\
\text { lus }\end{array}$ & $\begin{array}{l}0.76 \\
9^{*}\end{array}$ & $0.988^{*}$ & 1 & & & & $\begin{array}{l}1.00 \\
0^{* *}\end{array}$ & $0.931^{*}$ & 1 & & & \\
\hline $\begin{array}{l}\text { Tilapi } \\
\text { a zillii } \\
\end{array}$ & $\begin{array}{l}0.87 \\
8^{* * *} \\
\end{array}$ & $0.999^{*}$ & 0.981 & 1 & & & $\begin{array}{l}0.95 \\
8^{* *} \\
\end{array}$ & $0.996^{*}$ & 0.961 & 1 & & \\
\hline $\begin{array}{l}\text { Solea } \\
\text { vulga } \\
\text { ris } \\
\end{array}$ & $\begin{array}{l}\mathbf{0 . 8 8} \\
0^{* *} \\
\end{array}$ & $0.999^{*}$ & $\underset{* *}{0.980}$ & $\begin{array}{l}1.00 \\
0^{* * *} \\
\end{array}$ & 1 & & $\begin{array}{l}0.99 \\
8^{* *} \\
\end{array}$ & $0.950^{*}$ & $\underset{* *}{0.998}$ & $\begin{array}{l}0.97 \\
5^{* *} \\
\end{array}$ & 1 & \\
\hline \multirow[t]{2}{*}{$\begin{array}{l}\text { Ather } \\
\text { ina } \\
\text { sp. } \\
\end{array}$} & $\begin{array}{l}0.90 \\
4^{* *} \\
\end{array}$ & $0.995^{*}$ & $\underset{* *}{0.968}$ & $\begin{array}{l}0.99 \\
8^{* *} \\
\end{array}$ & $\underset{* *}{0.998}$ & 1 & $\begin{array}{l}0.98 \\
8^{* *}\end{array}$ & $0.974^{*}$ & ${ }_{* *}^{0.989}$ & $\begin{array}{l}0.99 \\
1^{* *} \\
\end{array}$ & 0.996 & 1 \\
\hline & \multicolumn{6}{|l|}{$\mathrm{Zn}$} & & & & & & \\
\hline $\begin{array}{l}\text { Wate } \\
\mathbf{r}\end{array}$ & 1 & & & & & & & & & & & \\
\hline $\begin{array}{l}\begin{array}{l}\text { Sedi } \\
\text { ment }\end{array} \\
\end{array}$ & $\begin{array}{l}0.94 \\
7^{* *}\end{array}$ & 1 & & & & & & & & & & \\
\hline $\begin{array}{l}\text { Mugil } \\
\text { cepha }\end{array}$ & \begin{tabular}{|l|l|}
0.82 \\
$0^{* * *}$ \\
\end{tabular} & $0.961^{*}$ & 1 & & & & & & & & & \\
\hline
\end{tabular}




\begin{tabular}{|c|c|c|c|c|c|c|}
\hline & 0.95 & $1.000^{*}$ & 0.956 & & & \\
\hline a zillii & $\mathbf{2}^{* * *}$ & & $* *$ & 1 & & \\
\hline $\begin{array}{l}\text { Solea } \\
\text { vulga } \\
\text { ris }\end{array}$ & $\begin{array}{l}0.91 \\
7^{* * 4}\end{array}$ & $0.997^{*}$ & 0.980 & $\begin{array}{l}0.99 \\
5^{* * *}\end{array}$ & 1 & \\
\hline $\begin{array}{l}\text { Ather } \\
\text { ina } \\
\text { sp. }\end{array}$ & $\begin{array}{l}0.95 \\
8^{* * *}\end{array}$ & $0.999^{*}$ & 0.949 & $\begin{array}{l}1.00 \\
0^{* * *}\end{array}$ & $\underset{* * *}{0.993}$ & 1 \\
\hline
\end{tabular}

Correlation coefficient significant at $* P \leq 0.05, * * P \leq 0.01$ and NS; nonsignificant.

Table (8): Compare between heavy metals in muscles of fishes species by two-way ANOVA (F-test)

\begin{tabular}{|c|c|c|c|c|c|c|c|c|c|c|c|c|c|c|}
\hline \multirow{3}{*}{$\begin{array}{l}\text { Source } \\
\text { s }\end{array}$} & \multicolumn{14}{|c|}{ Heavy metals } \\
\hline & \multicolumn{2}{|c|}{ Cd } & \multicolumn{2}{|c|}{$\mathbf{C u}$} & \multicolumn{2}{|c|}{$\mathrm{Fe}$} & \multicolumn{2}{|c|}{ Mn } & \multicolumn{2}{|c|}{$\mathbf{N i}$} & \multicolumn{2}{|c|}{$\mathbf{P b}$} & \multicolumn{2}{|c|}{$\mathbf{Z n}$} \\
\hline & $\begin{array}{l}\text { T- } \\
\text { test }\end{array}$ & $\begin{array}{l}P \text { - } \\
\text { val } \\
\text { ue }\end{array}$ & $\begin{array}{l}\text { T- } \\
\text { test } \\
\end{array}$ & $\begin{array}{l}P \text { - } \\
\text { val } \\
\text { ue }\end{array}$ & $\begin{array}{l}\text { T- } \\
\text { test }\end{array}$ & $\begin{array}{l}P \text { - } \\
\text { val } \\
\text { ue }\end{array}$ & $\begin{array}{l}\text { T- } \\
\text { test }\end{array}$ & $\begin{array}{l}P- \\
\text { val } \\
\text { ue }\end{array}$ & $\begin{array}{l}\text { T- } \\
\text { test }\end{array}$ & $\begin{array}{l}P- \\
\text { val } \\
\text { ue }\end{array}$ & $\begin{array}{l}\text { T- } \\
\text { test } \\
\end{array}$ & $\begin{array}{l}P- \\
\text { val } \\
\text { ue }\end{array}$ & $\begin{array}{l}\text { T- } \\
\text { test }\end{array}$ & $\begin{array}{l}P \text { - } \\
\text { val } \\
\text { ue }\end{array}$ \\
\hline Water & $\begin{array}{l}1.6 \\
22\end{array}$ & $\begin{array}{l}0.12 \\
2\end{array}$ & $\begin{array}{l}0.8 \\
43\end{array}$ & $\begin{array}{l}0.41 \\
0\end{array}$ & $\begin{array}{l}1.4 \\
04\end{array}$ & $\begin{array}{l}0.17 \\
7\end{array}$ & $\begin{array}{l}1.1 \\
09\end{array}$ & $\begin{array}{l}0.28 \\
2\end{array}$ & $\begin{array}{l}6.2 \\
18\end{array}$ & $\begin{array}{l}0.00 \\
0\end{array}$ & $\begin{array}{l}1.3 \\
89\end{array}$ & $\begin{array}{l}0.18 \\
2\end{array}$ & $\begin{array}{l}3.8 \\
45\end{array}$ & $\begin{array}{l}0.00 \\
1\end{array}$ \\
\hline $\begin{array}{l}\text { Sedim } \\
\text { ent }\end{array}$ & $\begin{array}{l}0.7 \\
15\end{array}$ & $\begin{array}{l}0.48 \\
3\end{array}$ & $\begin{array}{l}2.4 \\
63\end{array}$ & $\begin{array}{l}0.02 \\
4\end{array}$ & $\begin{array}{l}0.1 \\
18\end{array}$ & $\begin{array}{l}0.90 \\
8\end{array}$ & $\begin{array}{l}0.0 \\
63\end{array}$ & $\begin{array}{l}0.95 \\
1\end{array}$ & $\begin{array}{l}2.7 \\
54\end{array}$ & $\begin{array}{l}0.01 \\
3\end{array}$ & $\begin{array}{l}0.7 \\
18\end{array}$ & $\begin{array}{l}0.48 \\
2\end{array}$ & $\begin{array}{l}2.1 \\
27\end{array}$ & $\begin{array}{l}0.04 \\
8\end{array}$ \\
\hline
\end{tabular}

Table (9): Compare between heavy metals in spring and summer in water and sediment by independent samples (T-test)

\begin{tabular}{|c|c|c|c|c|c|c|c|c|c|c|c|c|c|c|}
\hline \multirow{3}{*}{$\begin{array}{l}\text { Spec } \\
\text { ies }\end{array}$} & \multicolumn{14}{|c|}{ Heavy metals } \\
\hline & \multicolumn{2}{|c|}{ Cd } & \multicolumn{2}{|c|}{$\mathbf{C u}$} & \multicolumn{2}{|c|}{$\mathbf{F e}$} & \multicolumn{2}{|c|}{ Mn } & \multicolumn{2}{|c|}{$\mathbf{N i}$} & \multicolumn{2}{|c|}{$\mathbf{P b}$} & \multicolumn{2}{|c|}{ Zn } \\
\hline & $\begin{array}{l}\text { F- } \\
\text { test }\end{array}$ & $\begin{array}{l}P \text { - } \\
\text { val } \\
\text { ue }\end{array}$ & $\begin{array}{l}\text { F- } \\
\text { test }\end{array}$ & $\begin{array}{l}P \text { - } \\
\text { val } \\
\text { ue }\end{array}$ & $\begin{array}{l}\text { F- } \\
\text { test }\end{array}$ & $\begin{array}{l}P \text { - } \\
\text { val } \\
\text { ue }\end{array}$ & $\begin{array}{l}\text { F- } \\
\text { test }\end{array}$ & $\begin{array}{l}P \text { - } \\
\text { val } \\
\text { ue }\end{array}$ & $\begin{array}{l}\text { F- } \\
\text { test }\end{array}$ & $\begin{array}{l}P \text { - } \\
\text { val } \\
\text { ue } \\
\end{array}$ & $\begin{array}{l}\text { F- } \\
\text { test }\end{array}$ & $\begin{array}{l}P \text { - } \\
\text { val } \\
\text { ue } \\
\end{array}$ & $\begin{array}{l}\text { F- } \\
\text { test }\end{array}$ & $\begin{array}{l}P \text { - } \\
\text { val } \\
\text { ue } \\
\end{array}$ \\
\hline Fish & $\begin{array}{l}13.0 \\
00\end{array}$ & $\begin{array}{l}0.0 \\
01\end{array}$ & $\begin{array}{l}513 . \\
860\end{array}$ & $\begin{array}{l}0.0 \\
00\end{array}$ & $\begin{array}{l}24.8 \\
33\end{array}$ & $\begin{array}{l}0.0 \\
00\end{array}$ & $\begin{array}{l}10.9 \\
05\end{array}$ & $\begin{array}{l}0.0 \\
03\end{array}$ & $\begin{array}{l}31.8 \\
79\end{array}$ & $\begin{array}{l}0.0 \\
00\end{array}$ & $\begin{array}{l}27.5 \\
29\end{array}$ & $\begin{array}{l}0.0 \\
00\end{array}$ & $\begin{array}{l}19.0 \\
68\end{array}$ & $\begin{array}{l}0.0 \\
00\end{array}$ \\
\hline
\end{tabular}
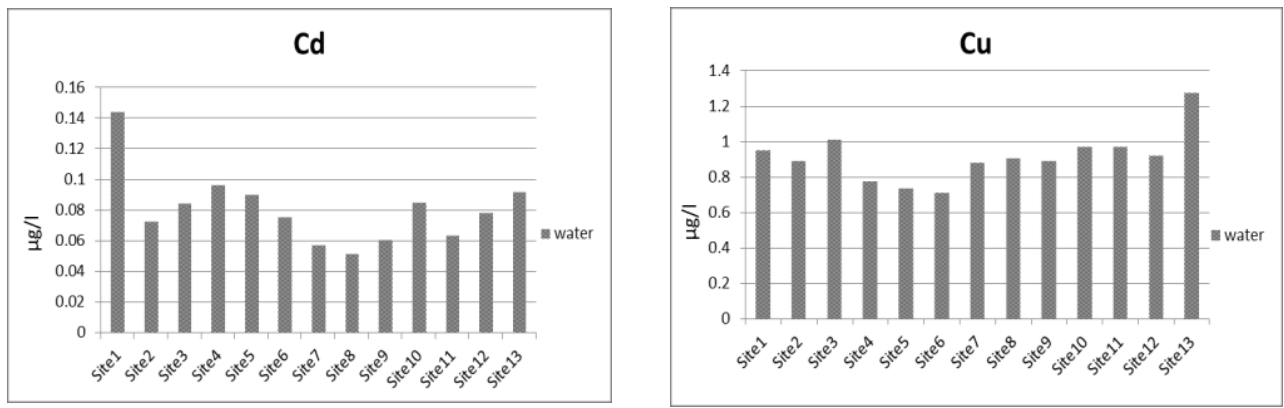

Figure (1): The annual concentrations ( $\mu \mathrm{g} / \mathrm{l})$ of $\mathrm{Cd}$ and $\mathrm{Cu}$ in water samples collected from Lake Quaron. 

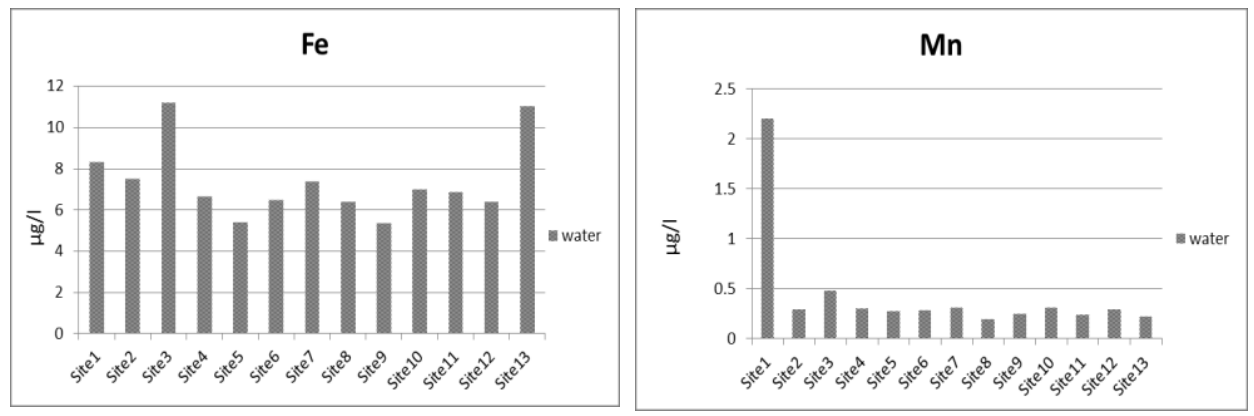

Figure (2): The annual concentrations ( $\mu \mathrm{g} / \mathrm{l})$ of Fe and Mn in water samples collected from Lake Quaron.
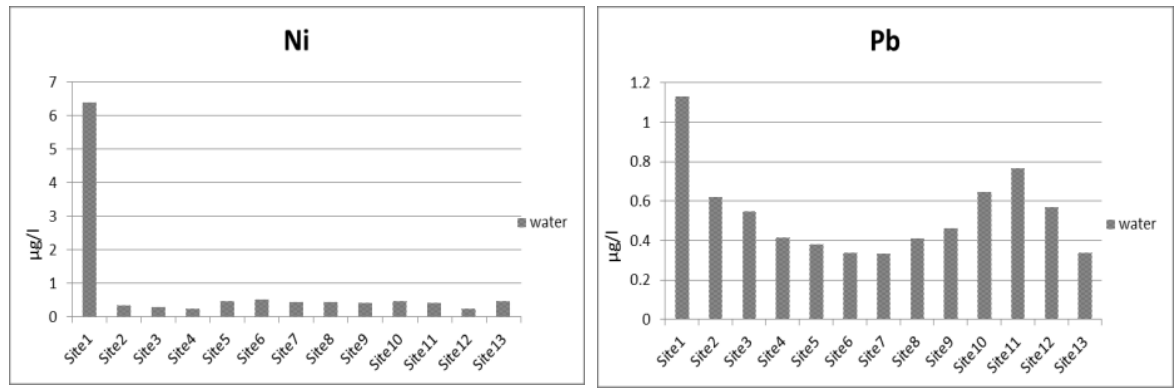

Figure (3): The annual concentrations ( $\mu \mathrm{g} / \mathrm{l})$ of $\mathrm{Ni}$ and $\mathrm{Pb}$ in water samples collected from Lake Quaron.
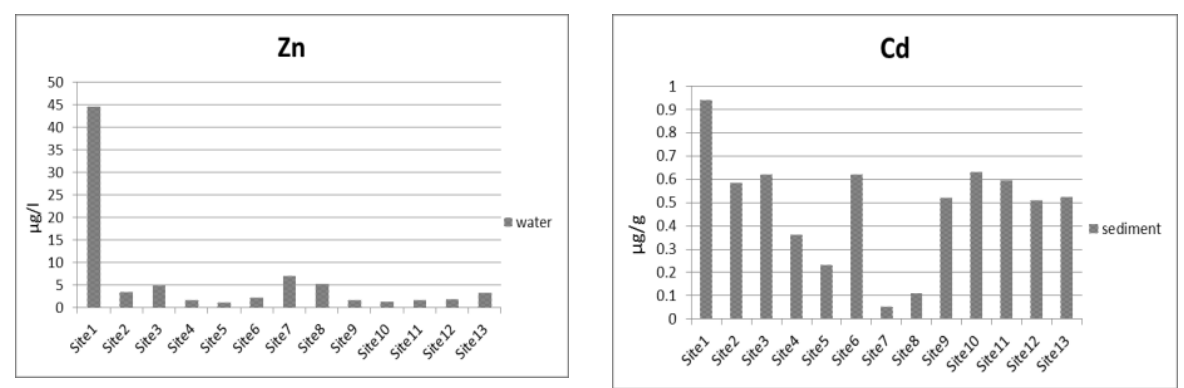

Figure (4): The annual concentrations $(\mu \mathrm{g} / \mathrm{l})$ of $\mathrm{Zn}$ in water samples collected from Lake Quaron.

Figure (5): The annual concentrations $(\mu \mathrm{g} / \mathrm{g})$ of $\mathrm{Cd}$ in sediment samples collected from Lake Quaron. 

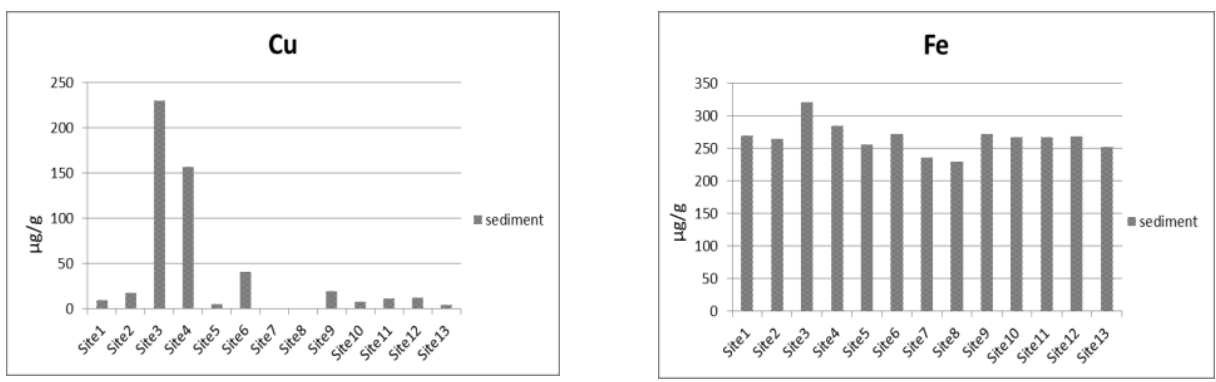

Figure (6): The annual concentrations $(\mu \mathrm{g} / \mathrm{g})$ of $\mathrm{Cu}$ and $\mathrm{Fe}$ in sediment samples collected from Lake Quaron.
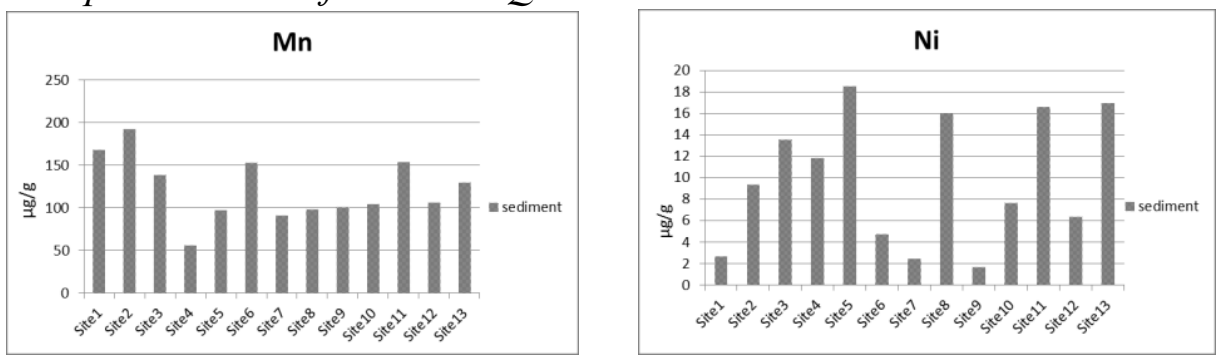

Figure (7): The annual concentrations $(\mu \mathrm{g} / \mathrm{g})$ of $\mathrm{Mn}$ and $\mathrm{Ni}$ in sediment samples collected from Lake Quaron.
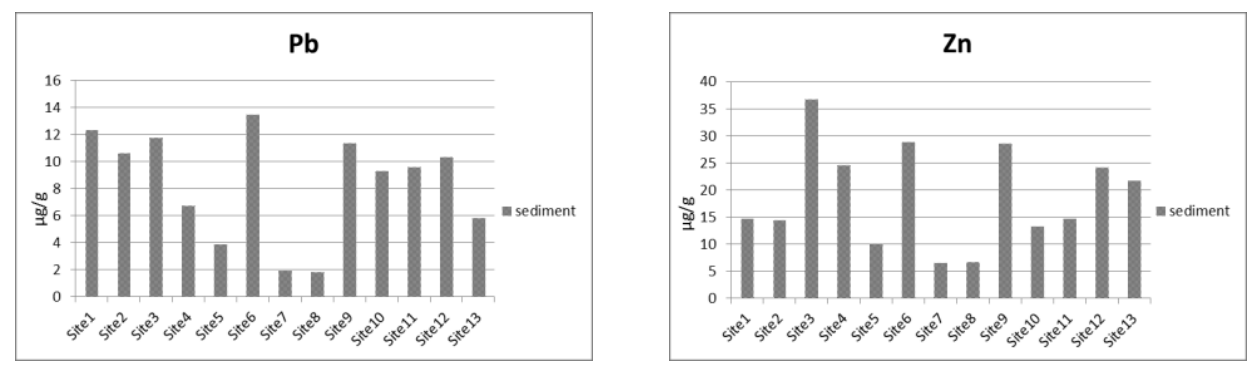

Figure (8): The annual concentrations ( $\mu \mathrm{g} / \mathrm{g})$ of $\mathrm{Pb}$ and $\mathrm{Zn}$ in sediment samples collected from Lake Quaron.
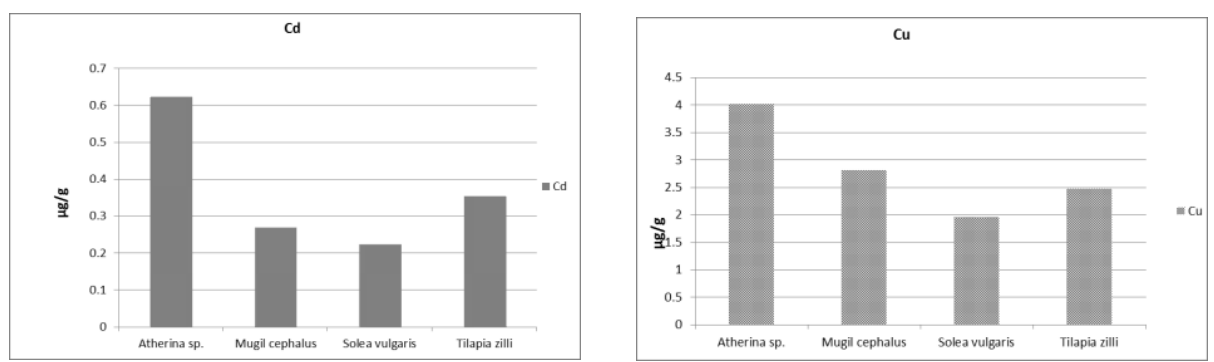

Figure (9): The average concentrations ( $\mu \mathrm{g} / \mathrm{g} d r y \mathrm{wt}$.) of $\mathrm{Cd}$ and $\mathrm{Cu}$ in fish samples collected from Lake Quaron. 

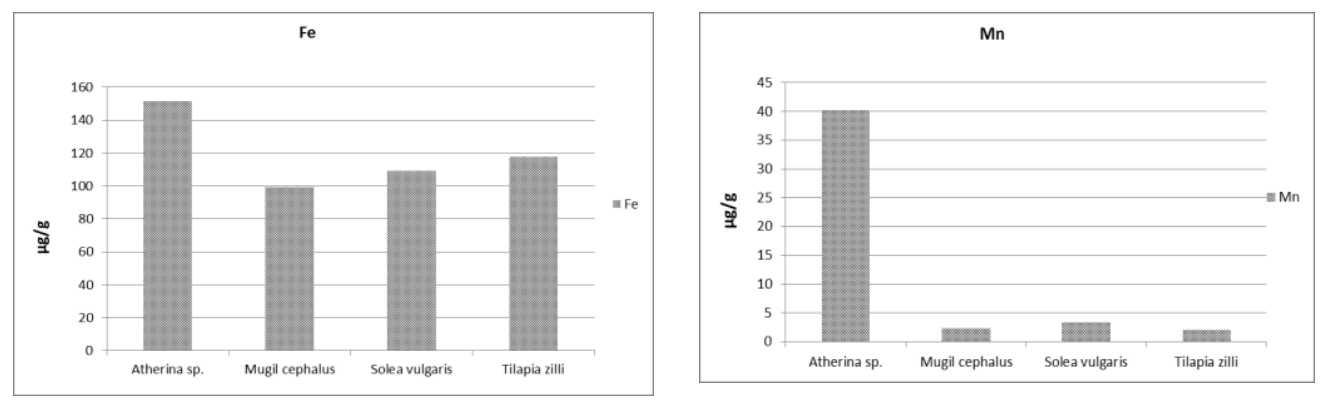

Figure (10): The average concentrations ( $\mu \mathrm{g} / \mathrm{g} d r y \mathrm{wt}$.) of $\mathrm{Fe}$ and $\mathrm{Mn}$, in fish samples collected from Lake Quaron.
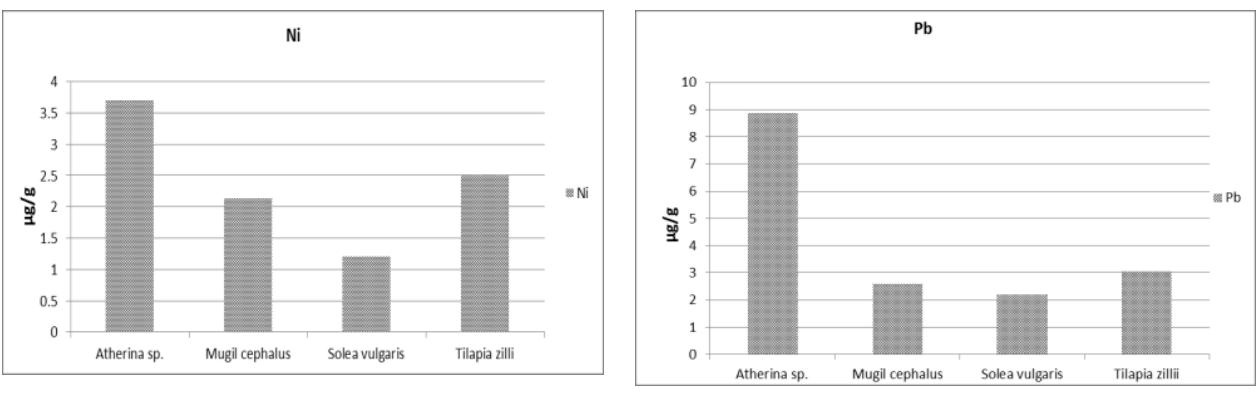

Figure (11): The average concentrations ( $\mu \mathrm{g} / \mathrm{g} d r y$ wt.) of $\mathrm{Ni}$ and $\mathrm{Pb}$ in fish samples collected from Lake Quaron.

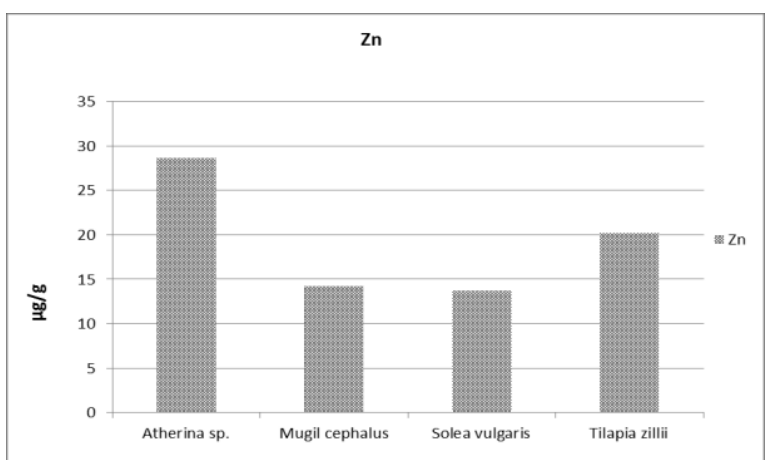

Figure (12): The average concentrations ( $\mu \mathrm{g} / \mathrm{g} d r \mathrm{y}$ wt.) of $\mathrm{Zn}$ in fish samples collected from Lake Quaron. 

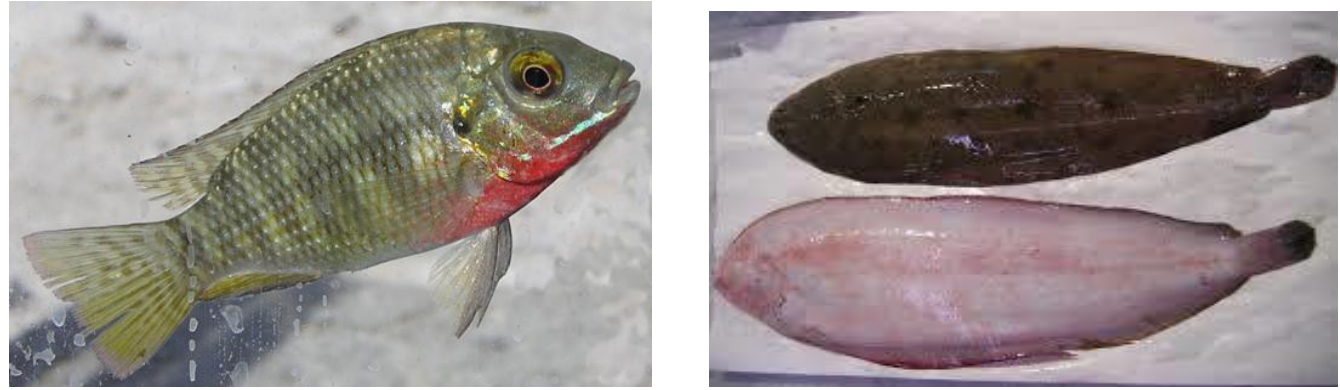

Photo (2): Tilapia (Tilapia zillii) Photo (3): Common Sole (Solea vulgaris)
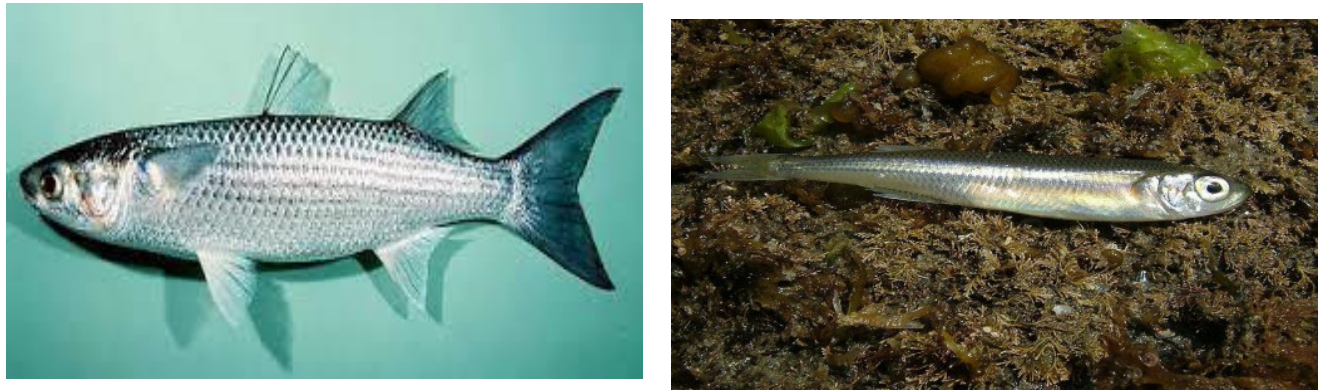

Photo (4): Flathead Mullet (Mugil cephalus)

Photo (5): Sand Smelt (Atherina presbyter)

\section{Discusion}

Heavy metal pollution is one of the five major types of toxic pollutants commonly present in surface waters. If they reach high enough levels, they may become toxic to aquatic organisms (Mason, 1991).

Lake Quaron was a fresh water lake then became a brackish water lake, as the lake is closed ecosystem and the main source of water is agricultural drainage water of ElFayoum Province. Highly evaporation of water leads to increases concentration of heavy metals, salts, pesticides and finally, leads to changes of the water quality and biodiversity (Ali et al, 2008).

In the present study, the annual average concentrations of the heavy metals in water were within the permissible limits according to USEPA (1986). The same order of concentrations were recorded by Abdel-Satar et al (2010) in order $\mathrm{Zn}>\mathrm{Cu}>\mathrm{Pb}>\mathrm{Cd}$, while Ibrahim and Ramzy (2013) recorded higher concentrations of $\mathrm{Fe}, \mathrm{Mn}, \mathrm{Pb}, \mathrm{Cu}$, $\mathrm{Cd}$, , and $\mathrm{Zn}$ than the concentrations of same metals in the present study . It is noticed that site (1) had the highest concentrations of $\mathrm{Cd}, \mathrm{Fe}$, $\mathrm{Mn}, \mathrm{Ni}, \mathrm{Pb}$ and $\mathrm{Zn}$ in water samples and that is related to presence of the 
pumping station (hydraulic pump that pumping the agricultural and sewage drainage that collected from the minor drains into lager drain namely Dayer El-Birka which transfers a part of wastewater to the lake by pumping station, this result agreed with Authman and Abbas (2007).

In comparison with the other lakes in Egypt, the water in the present study was showed lower levels of $\mathrm{Cd}, \mathrm{Cu}, \mathrm{Fe}, \mathrm{Mn}, \mathrm{Pb}$, and $\mathrm{Zn}$ than that recorded in Lake Edku (Masoud et al, 2005) and Lake Manzala (Salah-Eldein, 2012 and Bahnasawy et al, 2011).

In the present study, the annual average concentrations of the heavy metals in surface sediment samples which were collected from the study area were in the following order; $\mathrm{Fe}>\mathrm{Mn}>\mathrm{Zn}>\mathrm{Pb}>\mathrm{Cu}>\mathrm{Ni}>$ $\mathrm{Cd}$ and within the permissible limits of sediment quality guidelines (SQG) according to Persaud et al (1990) except $\mathrm{Cu}$ was higher. Unlike that, Abdel-Satar et al (2010) recorded the $\mathrm{Cd}$ level was exceeded the permissible limits of (SQG) while the levels of $\mathrm{Cu}, \mathrm{Fe}$, $\mathrm{Mn}, \mathrm{Pb}$ and $\mathrm{Zn}$ were within the SQG.

In the present study the levels of all estimated metals in the sediment of Lake Quaron were lower than that detected by Abdel-Satar et al (2010) and Ali and Fishar (2005) except $\mathrm{Cu}$ and $\mathrm{Fe}$ were higher.

In comparison with the other lakes in Egypt, the sediment in the present study was showed lower levels of metals than that recorded in Lake Edku (Masoud et al, 2005) except $\mathrm{Cu}, \mathrm{Ni}$ and $\mathrm{Pb}$ were higher in Quaron Lake.

It was noticed that the most of levels of metals in sediment were higher than that estimated in the water in same sites and these results agreed with Saeed and Shaker (2008), who reported that lake sediments are normally the final pathway of both natural and anthropogenic components produced or derived to the environment.

The organic pollutants and heavy metals present in water tend to be concentrated in sediment. Also agreed with Silva and Rezende (2002) who reported that lake sediments can serve as archives of environmental changes through time.

Generally the eastern sites of water and sediment samples of Lake Quaron had higher concentrations of metals than the western sites and these results in agreement with $\boldsymbol{A l i}$ and Fishar (2005) who stated that the eastern sites of the lake generally, had more concentrations of heavy metals than the western sites and that may related to presence of El-Batts drain and pumping stations.

Therefore the obtained results in this work may reflect that the sediment of Lake Quaron is the final reservoir for most heavy metals, gives a good indication to the environmental changes, consider as an alarm to the toxic 
effect in this ecosystem and this may due to this Lake receives heavy loads of organic and inorganic pollutants via several agricultural drains and huge amounts of raw sewage, agricultural and industrial wastewater discharged into the Lake and this agreed to that recorded by Badawy et al ( 1995) and Abdel-Moati and El-Sammak (1997).

In the present study site (7) and site (8) which located north western of the lake were generally had lower annual levels of most metals in both water and sediment samples than other sites as both sites located away from direct or indirect sources of pollution as El-Batts Drain, ElWadi Drain, pumping stations, agricultural and touristic activities in the southern coast of Lake Quaron ,also movement and directions of water waves in Lake Quaron as it directed from north western to south eastern part of the lake ( from high density to low density water) and these results agreed with El Helessy (2011).

In the present study $\mathrm{Cu}$ generally was higher and that is due to presence of About (615) woody non-motorized fishing boats operating in the lake of Quaron (ElSerafy et al, 2014) that using antifouling coatings which are the major source of $\mathrm{Cu}$ pollution (Ghanem, 1986).

In estimation the heavy metals levels in Atherina $s p$. were in the following order; $\mathrm{Fe}>\mathrm{Mn}>\mathrm{Zn}>\mathrm{Pb}>$ $\mathrm{Cu}>\mathrm{Ni}>\mathrm{Cd}$, Mugil cephalus were in the following order; $\mathrm{Fe}>$ $\mathrm{Zn}>\mathrm{Cu}>\mathrm{Pb}>\mathrm{Mn}>\mathrm{Ni}>\mathrm{Cd}$, Solea Vulgaris were in the following order; $\mathrm{Fe}>\mathrm{Zn}>\mathrm{Mn}>\mathrm{Pb}>\mathrm{Cu}>\mathrm{Ni}>$ $\mathrm{Cd}$ and Tilapia zillii were in the following order; $\mathrm{Fe}>\mathrm{Zn}>\mathrm{Pb}>\mathrm{Ni}$ $>\mathrm{Cu}>\mathrm{Mn}>\mathrm{Cd}$ in the present study . From the obtained results of levels of heavy metals in fishes were in the following order ;Atherina sp.> Tilapia zillii $>$ Mugil cephalus $>$ Solea Vulgaris and this result agreed with Authman and Abbas (2007) who reported that the concentration of heavy metals in the fish samples indicated that Tilapia zilli were higher than those of Mugil cephalus which is attributed to their feeding behavior, and disagreed with Mansour and Sidky (2002) who stated that levels of heavy metals in Mugil cephalus were higher than those of Tilapia zilli. Also disagreed with Ali and Fishar (2005) who reported that heavy metals concentration in the fish species were in the following order; Solea sp. $>$ Mugil sp. > Tilapia sp.

Generally the levels of heavy metals of $\mathrm{Fe}, \mathrm{Mn}, \mathrm{Ni}$, and $\mathrm{Pb}$ in all fish species were higher than the permissible limits except $\mathrm{Fe}$ in Mugil cephalus was within the permissible limits according to WHO (1989) and FAO (1983), while the levels of $\mathrm{Cd}, \mathrm{Cu}$ and $\mathrm{Zn}$ within the permissible limits, this result agreed with Mansour and Sidky (2002) who stated that among the analyzed metals (e.g. $\mathrm{Zn}, \mathrm{Fe}$, $\mathrm{Mn}, \mathrm{Cu}$, cadmium , $\mathrm{Cr}, \mathrm{Ni}, \mathrm{Pb}, \mathrm{Co}$ and $\mathrm{Sn}$ ) were found in fish at mean 
concentrations above the permissible limits proposed by FAO.

In comparable to levels of heavy metals in fish and water samples that were collected from lake Quaron, it is noticed that all levels of heavy metals in fish species were higher than that levels estimated in water and that is agreed with Gomaa (1995) who documented that fish has large amounts of some metals such as lead and cadmium due to presence of fish generally, at the top of the aquatic food chain, in turn causes health hazards to humans who consumed this fish. Also agreed with Silveira et al (2004), Elghobashy et al (2001), MaCarthy and Shugart (1990) Shakweer (1998) who concluded that the degree fish pollution depending on the degree of pollution in the aquatic environment where fish living.

In comparison with the other lakes in Egypt, generally the fish species in the present study were showed higher levels of $\mathrm{Cd}, \mathrm{Cu}, \mathrm{Fe}, \mathrm{Mn}, \mathrm{Pb}$, and $\mathrm{Zn}$ than that recorded in Oreochromis niloticus in Lake Edku and Borollus (Saeed and Shaker, 2008).

The metals of $\mathrm{Cd}, \mathrm{Cu}, \mathrm{Fe}, \mathrm{Mn}, \mathrm{Ni}$, $\mathrm{Pb}$ and $\mathrm{Zn}$ contents in water were significantly $(\mathrm{P} \leq 0.01)$ and positively correlated with $\mathrm{Cd}, \mathrm{Cu}$, $\mathrm{Fe}, \mathrm{Mn}, \mathrm{Ni}, \mathrm{Pb}$ and $\mathrm{Zn}$ contents respectively in sediment, fish sp. these results agreed with Saeed and Shaker (2008) who concluded that Sediment concentrate the heavy metals so it considered as a good indicator for water pollution. Also agreed with Gomaa (1995) who documented that fish are often at the top of the aquatic food chain so, concentrate large amounts of some metals.

The metals of $\mathrm{Cd}, \mathrm{Cu}, \mathrm{Fe}, \mathrm{Mn}, \mathrm{Ni}$, $\mathrm{Pb}$ and $\mathrm{Zn}$ content in fishes were significantly influenced $(\mathrm{P} \leq 0.05)$ by species these result agreed with Authman and Abbas (2007) who reported that the concentration of heavy metals in the fish samples showed that Mugil cephalus were lower than Tilapia zilli those of which is attributed to their feeding behavior. These results agreed with Ali et al (2008) who reported that extensive evaporation of water from Lake Quaron increases concentration of heavy metals, leading to changes in the water quality of and biodiversity. Also agreed with Silva and Rezende (2002) who reported that lake sediments can serve as archives of environmental changes through time.

\section{References}

Abdel-Moati, M. A. and ElSammak, A. A. (1997): Man-made impact on the geochemistry of the NileDeltaLakes. A study of metals concentrations in sediments. Water, Air and Soil Pollution. 97: 413-429.

Abdel-Satar, A.M., Goher, M.E. and Sayed, M.F.(2010): Recent Environmental Changes in Water and Sediment Quality of Lake 
Quaron ,Egypt. J. of Fisheries and Aquatic Sci. 5 (2):56-69.

Adeloju,S.B., Dhindsa, H. S. and Tandon, R. K. (1994): Evaluation of some wet decomposition methods for mercury determination in biological and environmental materials by cold vapour atomic absorption spectrometry. Analyt. Chem. Acta., 285: 359-364.

Al-Ghais, S.M.(1995): heavy metals concentration in the tissue sparus sebra from the united Arab Emirates. Bull. Eniviron. Contam. Toxicol. 55, pp:581.

Ali, M.H.H. and Fishar, M.R.A. (2005): Accumulation of trace metals in some benthic invertebrate and fish species revelant to their concentration in water and sediment of Lake Quaron ,Egypt. Egypt. J. of Aqua. Research Vol. 31, No.1.

Ali, F.Kh., El-Shafai, S.A., Samhan, F.A. and Khalil, W.K.B.(2008): Effects of water pollution on expression of immune response genes of Soleaaegyptiacain Lake Quaron . African J. Biotech. 7(10):1418-1425. American Public Health Association (A.P.H.A) (1985): Standard Methods for Examination of Water and Waste Water. $16^{\text {th }}$ Edition .Washington, D.C.

Authman, M.M.N. and Abbas, H.H.H. (2007): Accumulation and distribution of copper and zinc in both water and some vital tissues of two fish species (Tilapia zilli and Mugil cephalus) of Lake Quaron , Fayoum Province, Egypt. Pakistan J. Biol. Sci. 10(13):2106-2122.
Badawy, M.I.; Wahaab, R.A. and AbouWaly, H.F. (1995): Petroleum and chlorinated hydrocarbons in water from Lake Manzala and associated canals. Bull. Environ. Contam. Toxicol. 55: 258-263.

Bahnasawy, M.; Khidr, A. and Nadia, D. (2011): Assessment of heavy metal concentrations in water, plankton, and fish of Lake Manzala, Egypt. Turk J Zool, 35(2): 271-280. Brewer, P. G., Spencer, D.W. and Smith, C. L. (1969): Determination of trace metals in seawater by atomic absorption spectrophotometry. Atomic Absorption Spectroscopy, (ASTM STP), American Society for Testing and Materials 443: 70-77.

El Helessy, F.M.R. (2011): Lake Quaron Area, Study of Applied Geomorphology. M.Sc. thesis, El Azhar Univ. Fac. of Humanity Studies Geographical Department, 199-202.

Elghobashy, A.H., Zaghloul, K.H., and Metwally, M.A. (2001): Effect of some water pollutants on the Nile Tilapia, Oreochromis niloticus collected from the river Nile and some Egyptian Lakes, Egypt, J. Aquat. Biol. Fish.,5(4):251-279.

El-Serafy, S.S., El-Haweet, A.A., El-Ganiny, A., and El-Far, A.M.(2014): Quaron Lake fisheries; Fishing gears, Species composition and Catch per unit effort. Egypt. J. Aquat. Biol. \& Fish., Vol. 18, No. 2: 39- 49 .

FAO (1983): Compilation of legal limits for hazardous substances in 
fish and fishery products. FAO Fishery Circular, No. 464, 5-100.

Ghanem, N. A. (1986): the problem of marine pollution by antifouling coatings and potential solutions. In: Marine environment and pollution, Proc. Of the $1^{\text {st }}$ Arabian Gulf Confr. On Environment and Pollution Kuwait, 7-9 Feb. 1982 eds. R. Halwagy, D. Clayton, and M. Behbehani., Kuwait Univ., Fac. Sci., KFAS and EPL. Kuwait, 157-169.

Gomaa, M.N.E. (1995): Recycling study of some heavy metals in the Egyptian aquatic ecosystem Food Chem., 54: 297-303.

Ibrahim, L.A. and Ramzy, E.M. (2013): Water quality and its impact on Tilapia zilli (Case Study) Quaron Lake -Egypt. International Water Tech.J.3: 170-191.

Khayatzadeh, J.andAbbasi, E. (2010): The effect of heavy metals on aquatic animals . The $1^{\text {st }}$ .International Applied Geological Congress ,Depart. of Geol. ,Islamic Azad Univ. Mashsad Branch ,Iran ,26-28 april.

MaCarthy J.F. and Shugart L.R.(1990): Biomarkers of environmental contamination . Lewis Publishers. New York . 475pp.

Mansour, S.A. and Sidky, M. M. (2002): Ecotoxicological studies.3. Heavy metals contaminating water and fish from Fayoum Governorate, Egypt . Food Chem.,78: 154-22.

Mason, C. F. (1991). Biology of fresh water pollution, 2nd end., Longman. New York.
Masoud, M.S., Elewa, A.A., Ali,A.E. and Mohamed, E.A.(2005): Distribution of some metals concentrations in water and sediments of Lake Edku ,Egypt. Bulletin of the Chemists and Technologists of Macedonia, Vol. 24, No. 1, pp. 21-34 (2005).

Mohamed, F.A.S. and Gad, N.S. (2008): Environmental pollution induced biochemical changes in tissues of Tilapia zillii, Solae vulgaris and Mugil capito from Lake Quaron, Egypt. Global Vet.2 (6): 327-336.

Persaud, D., Jaagumagi, R. and Hayton, A. (1990): The provincial sediment quality guidelines. Ontario Ministry of the Environment.

Saeed, S.M. and Shaker, I.M. (2008): Assesment of the heavy metals in the water and sediments and their effect on Oreochromis niloticus in the northern Delta lakes, Egypt. $8^{\text {th }}$ International Symposium on Tilapia in Aquaculture :475-490.

Salah-Eldein,

A.

M.(2012):Resident wild birds as bio-indicator for some heavy metals pollution in Lake Manzala M. V. Sc. Thesis in Wildlife Management and Zoo Medicine Department. Fac. Of Vet. Medicine ,Suez Canal Univ.

Shakweer, L.M. (1998): Concentration levels of some trace metals in Oreochromis niloticus at highly and less polluted areas of Mariut Lake. J. Egypt. Ger. Soc. Zool. 25: 125-144.

Silva, M. A. L. and Rezende, C. E. (2002). Behavior of selectedmicro 
and trace elements and organic matter in sediments of a fresh water system in South-East Brazil. The Science of the Total Environment, 292, 121-128.

Silveira-Cohignya, R., PrietoTrujillo, A. and Ascencio-Valleb, F. (2004): Effectsof different stressors in hematologicalvariables in cultured Oreochromisaureus. comparativeBioch.

Snedecor G, Cochran W, Cox D. (1989): Statistical Methods8th edition). The Iowa State University Press.

USEPA (United States Environmental Protection agency), (1986): Quality Criteria for Water. EPA 440/5-86-001. May 1986. Office of water regulations and standards. Washington DC., USA.

WHO (World Health Organization), (1989): Heavy metals-environmental aspects. Environment Health Criteria. No. 85. Geneva, Switzerland

\section{الملخص العربي}

تعتبر تركيزات المعادن الثقبلة الموجودة في البيئة ذو اهميه كبيرة وذللك لتأثيراتها الخطيرة علي

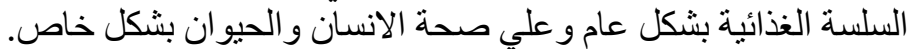

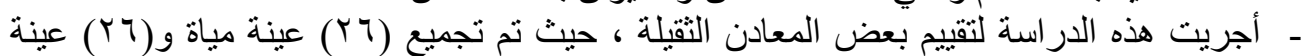

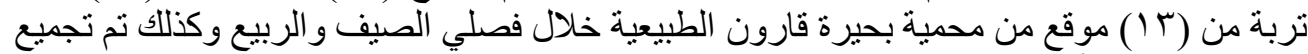

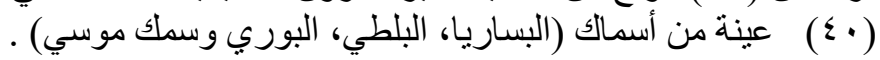

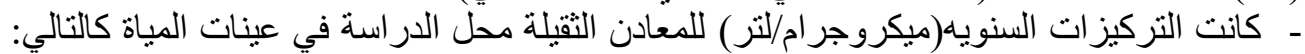

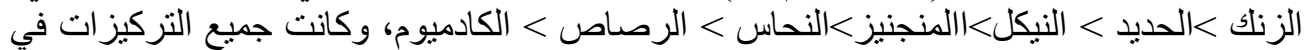

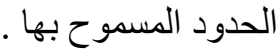

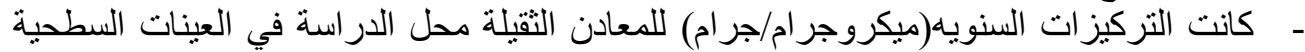

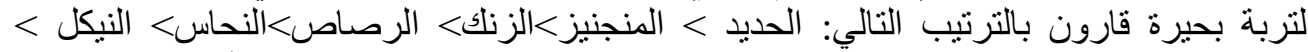

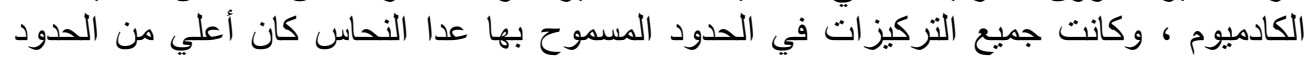

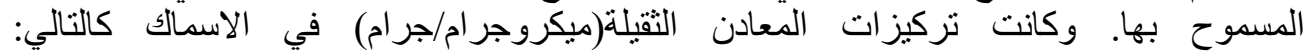
البساريا>>لبلطي >البوري>الموسي ز ـ ـ مما سبق فقد كثفت لنأ الدر اسة أن بحيرة قارون تعاني من مشكلة تلوث خطيرة. 